\title{
A Study of the Controlled Motion of a Four-wheeled Mecanum Platform
}

\section{B. I. Adamov}

The object of the study is the mobile platform of the KUKA youBot robot equipped with four Mecanum wheels. The ideal conditions for the point contact of the wheels and the floor are considered. It is assumed that the rollers of each Mecanum wheel move without slipping and the center of the wheel, the center of the roller axis, and the point of contact of the roller with the floor are located on the same straight line. The dynamics of the system is described using Appel's equations and taking into account the linear forces of viscous friction in the joints of the bodies. An algorithm for determination of the control forces is designed. Their structure is the same as that of the reactions of ideal constraints determined by the program motion of the point of the platform. The controlled dynamics of the system is studied using uniform circular motion of the platform point as an example: conditions for the existence and stability of steady rotations are found, conditions for the existence of stable-unstable stationary regimes and rotational motions of the platform are obtained. Within the framework of the theory of singular perturbations, an asymptotic analysis of the rotation of the platform is carried out.

Keywords: omniwheel, Mecanum wheel, omnidirectional platform, servo-constraint, youBot, singular perturbation

Received January 16, 2018

Accepted May 23, 2018

This work was supported by the RFBR grant no. 16-01-00429.

Boris I. Adamov

adamoff.b@yandex.ru

National Research University "Moscow Power Engineering Institute"

ul. Krasnokazarmennaya 14, Moscow, 111250 Russia

RUSSIAN JOURNAL OF NONLINEAR DYNAMICS, 2018, 14(2), 265-290 


\section{Introduction}

The application fields of mobile devices equipped with omniwheels are multiple and include working under constrained conditions of factory workshops and warehouses, logistic operations, special operation vehicles for disabled people, etc. [26].

In this paper we consider a mobile device equipped with omniwheels of Mecanum type (Ilon wheels, or Swedish wheels). A Mecanum wheel has a series of rollers placed at an angle around its periphery. The angle between each roller axis and the central wheel axis is $45^{\circ}[27,28]$.

One of the devices of this type is a youBot mobile platform produced by the KUKA Company [37]. It is an omnidirectional platform equipped with Mecanum wheels and one or two manipulators. The software on this robot is free, which permits one to make use of youBot for a wide range of research problems and academic pursuits.

There are a great number of publications on mechanics and control of omnidirectional platforms, including those with Mecanum wheels. Let us consider some of them.

Problems of the dynamics, optimal and adaptive motion control of omnidirectional platforms are analyzed in $[18,29,31,32,35]$. A number of publications are concerned with the mechanics of mobile devices with an arbitrary number, type and configuration of wheels $[4,8,9]$. The results of exact integration of dynamic equations and of research on steady motions of a three-wheeled mobile omniplatform are presented in $[18,19]$.

Omnidirectional wheels of various types are used for controlling the motion of spherical robots and spherical wheels. The motion of such systems is investigated in [4, 10, 30, 34].

We notice that, in the above publications devoted to the problems of mechanics and control of mobile omnidirectional platforms, friction forces acting on power transmission shafts and axes of the wheels are either ignored or grouped with control torques. In fact, in the above-mentioned publications, no attention is given to the consideration of frictional forces in the supports of wheel rollers.

In this work, we use the results of the theory of systems with servo-constraints for implementation of the program motion of the platform. Program motions (servo-constraints) can be realized by adjustment of forces within the framework of this theory [3, 11, 12]. In this case the structure of control actions repeats the structure of the reaction of ideal constraints, the equations of which coincide with the equations of program motion. Extremal properties of constraints resulting from the dynamic laws that prevail in mechanics make this control method somewhat optimal [15]. Examples of application of this method to robotics problems can be found in $[2,17,20-22,36]$.

The behavior of the system as a whole can be rather nontrivial (see the results of modeling in [2]) when the above method of executing program motion is used. Conclusions concerning the change in the motion pattern of the system due to the change in its mechanical energy were drawn already by H. Béghin [3, p. 29]. In the work of V. V. Kozlov [12, 13] it is shown that the dynamics of systems with servo-constraints is richer and more various than the dynamics of nonholonomic systems.

\section{The system under study and its model}

The mechanical system under study is an omnidirectional platform equipped with two pairs of Mecanum wheels (bearing rollers).

The above system simulates a youBot mobile robot by the KUKA Company (see Fig. 1). The values of geometrical and physical parameters of the system, which are used below, are taken 


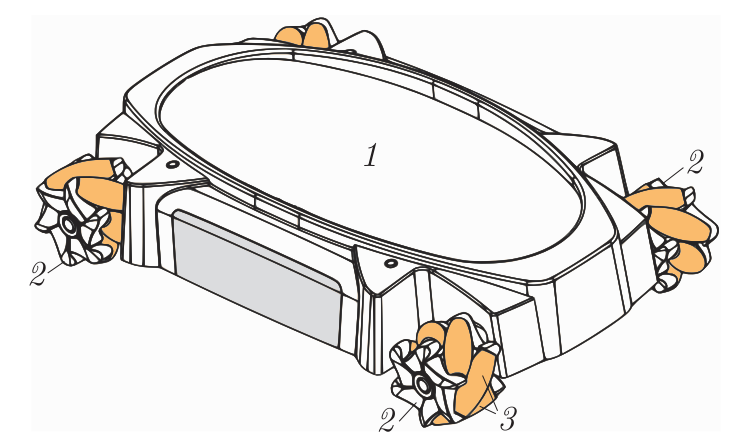

Fig. 1. Mobile omnidirectional platform of the youBot robot.

from the technical data of the robot [37]. Some parameters are estimated from the experimental data processing [1].

The mobile platform 1 of the youBot robot is equipped with four Mecanum wheels 2 (see Fig. 1). Each wheel has six symmetric bodies of revolution which are rollers 3 placed at an angle around its periphery. The angle between the roller axes and the wheel plane is $45^{\circ}$.

For all four wheels to have contact with the support while moving on a rough surfaces, youBot is equipped with a simple suspension of the front wheel pair, and its axis can rotate about the longitudinal axis of the platform through a restricted angle.

The motion of the platform on a horizontal plane is considered. It is assumed that the rollers continuously make contact with the underlying surface without loss of contact and without slipping, and the position of the contact point on the meridian of the roller does not depend on the rotation angle of the wheel. The platform and the equipment carried are considered as one rigid body.

\subsection{Kinematics of the platform of the youBot robot}

In order to describe the kinematics of a mobile robot, we introduce a fixed coordinate system $x y z$ with a horizontal plane $x y$. We also introduce a moving coordinate system $O X Y Z$ with origin at the geometric center of the platform $O$. The axis $O X$ is a longitudinal symmetry axis of the platform $O$, and the $O Y$ axis is a transverse axis. The coordinate axes $z$ and $O Z$ are vertical and aligned with each other. Further, coordinate representations of kinematic vector variables are given with respect to $O X Y Z$.

The position of the platform is defined by the Cartesian coordinates $x_{O}$ and $y_{O}$ of its geometric center $O$ in the fixed coordinate system $x y z$ and by the angle $\psi$ between the fixed axis $x$ and the $O X$ axis (see Fig. 2). We denote the wheel rotation angle $i(i=\overline{1,4})$ with respect to the robot body by $\varphi_{i}$, and the rotation angle of the contacting roller with respect to the wheel by $\gamma_{i}$ (see Fig. 2).

The motion of the platform is thus specified by the 11-dimensional vector of the generalized coordinates

$$
\boldsymbol{q}=\left(\begin{array}{lllllllllll}
x_{O} & y_{O} & \psi & \varphi_{1} & \varphi_{2} & \varphi_{3} & \varphi_{4} & \gamma_{1} & \gamma_{2} & \gamma_{3} & \gamma_{4}
\end{array}\right)^{\mathrm{T}}
$$

In the kinematic scheme of the platform shown in Fig. 2 the rollers of the wheels 1, 2, 3 and 4 contacting with the underlying surface are represented by ovals; $h$ denotes half the distance between the axes of the wheel pairs, and $l$ denotes half the distance between the contact points 


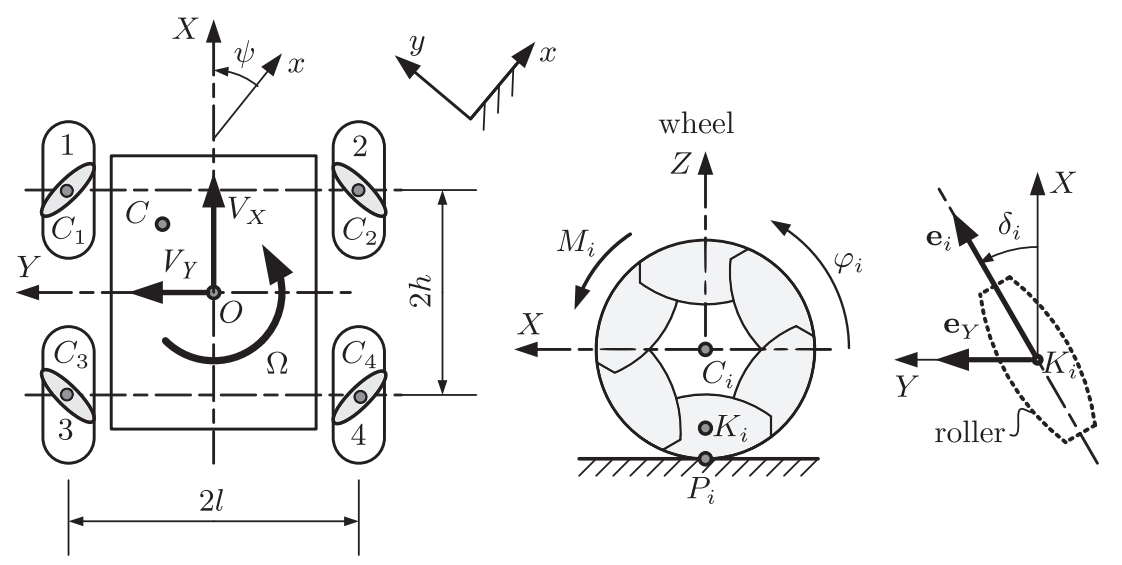

Fig. 2. Kinematic scheme of the mobile platform of the youBot robot.

of the opposite wheels. The radius of the outer surface of the Mecanum wheel is $C_{i} P_{i}=R$, and the radius of the cross section of the roller at the contact point is $K_{i} P_{i}=r$.

We assume the contact between the Mecanum wheels and the underlying surface to be ideal: the rollers move without loss of contact with the surface and without slipping, and the center $C_{i}$ of the $i$ th wheel, the center of the axis of the roller $K_{i}$, and the contact point $P_{i}$ between the roller and the floor are on the same straight line at each instant of time (see Fig. 2).

In the problem considered here the condition of no slipping of the rollers defines eight independent nonholonomic constraints. We describe the motion of the platform in pseudovelocities constituting the vector

$$
\dot{\pi}=\left(\begin{array}{lll}
V_{X} & V_{Y} & \Omega
\end{array}\right)^{\mathrm{T}},
$$

where $V_{X}, V_{Y}$ are the projections of the velocity vector of the point $O$ onto the corresponding moving axes and $\Omega$ is the angular velocity of the platform:

$$
V_{X}=\dot{x}_{O} \cos \psi+\dot{y}_{O} \sin \psi, \quad V_{Y}=-\dot{x}_{O} \sin \psi+\dot{y}_{O} \cos \psi, \quad \Omega=\dot{\psi} .
$$

Let us express the velocity vector of the point $P_{i}$ of contact of the roller with the underlying surface as

$$
\boldsymbol{v}_{P_{i}}=\boldsymbol{v}_{O}+\left[\boldsymbol{\Omega}, \boldsymbol{r}_{O P_{i}}\right]+\left[\dot{\varphi}_{i} \boldsymbol{e}_{Y}, \boldsymbol{r}_{C_{i} P_{i}}\right]+\left[\dot{\gamma}_{i} \boldsymbol{e}_{i}, \boldsymbol{r}_{K_{i} P_{i}}\right],
$$

where $\boldsymbol{v}_{O}=\left(V_{X}, V_{Y}, 0\right)^{\mathrm{T}}$ is the velocity vector of the geometric center of the platform $O ; \boldsymbol{r}_{C_{i} P_{i}}=$ $=(0,0,-R)^{\mathrm{T}}, \boldsymbol{r}_{K_{i} P_{i}}=(0,0,-r)^{\mathrm{T}} ; \rho_{X i}$ and $\rho_{Y i}$ are the coordinates of the center of the $i$ th wheel; $\boldsymbol{e}_{i}=\left(\cos \delta_{i}, \sin \delta_{i}, 0\right)^{\mathrm{T}}$ is the unit vector for the axis of the contacting roller of the $i$ th the axis being deviated by the angle $\delta_{i}$ from $O X$.

In accordance with the kinematic scheme (Fig. 2)

$$
\begin{gathered}
\delta_{1}=-45^{\circ}, \quad \rho_{X 1}=h, \quad \rho_{Y 1}=l ; \quad \delta_{2}=45^{\circ}, \quad \rho_{X 2}=h, \quad \rho_{Y 2}=-l ; \\
\delta_{3}=45^{\circ}, \quad \rho_{X 3}=-h, \quad \rho_{Y 3}=l ; \quad \delta_{4}=-45^{\circ}, \quad \rho_{X 4}=-h, \quad \rho_{Y 4}=-l .
\end{gathered}
$$

Making necessary transformations, we obtain from the ideal contact condition

$$
\boldsymbol{v}_{P_{i}}=\left(V_{X}-\Omega \rho_{Y i}-\dot{\varphi}_{i} R-\dot{\gamma}_{i} r \sin \delta_{i}, V_{Y}+\Omega \rho_{X i}+\dot{\gamma}_{i} r \cos \delta_{i}, 0\right)^{\mathrm{T}}=0 .
$$


From this we obtain expressions for the relative angular velocities of rotation of the wheels and the rollers:

$$
\begin{gathered}
\dot{\varphi}_{i}=\frac{1}{R}\left[V_{X}+V_{Y} \operatorname{tg} \delta_{i}+\left(\rho_{X i} \operatorname{tg} \delta_{i}-\rho_{Y i}\right) \Omega\right], \quad \dot{\gamma}_{i}=-\frac{V_{Y}+\Omega \rho_{X i}}{r \cos \delta_{i}}, \\
i=\overline{1,4} .
\end{gathered}
$$

Let us write the relations obtained in the vector-matrix form:

$$
\begin{gathered}
\dot{\boldsymbol{\varphi}}=\boldsymbol{C}_{\varphi} \dot{\boldsymbol{\pi}}, \quad \dot{\varphi}=\left(\begin{array}{c}
\dot{\varphi}_{1} \\
\dot{\varphi}_{2} \\
\dot{\varphi}_{3} \\
\dot{\varphi}_{4}
\end{array}\right), \quad \boldsymbol{C}_{\varphi}=\frac{1}{R}\left(\begin{array}{ccc}
1 & -1 & -h-l \\
1 & 1 & l+h \\
1 & 1 & -h-l \\
1 & -1 & l+h
\end{array}\right) ; \\
\dot{\gamma}=\boldsymbol{C}_{\gamma} \dot{\boldsymbol{\pi}}, \quad \dot{\boldsymbol{\gamma}}=\left(\begin{array}{c}
\dot{\gamma}_{1} \\
\dot{\gamma}_{2} \\
\dot{\gamma}_{3} \\
\dot{\gamma}_{4}
\end{array}\right), \quad \boldsymbol{C}_{\gamma}=\frac{\sqrt{2}}{r}\left(\begin{array}{ccc}
0 & 1 & h \\
0 & 1 & -h \\
0 & 1 & -h
\end{array}\right) .
\end{gathered}
$$

\subsection{Dynamics of the mobile platform}

Let us write the equations of motion for the mobile platform of the youBot robot in pseudovelocities $V_{X}, V_{Y}$ and $\Omega$. We neglect the mass of the rollers and of the rotating elements of the wheel drive power transmissions. The robot is considered as a system of perfectly rigid bodies. We assume the friction forces to be linear in velocities.

To obtain the equations of motion for the system, we make use of the Appel formalism $[6,14,16,23]$ :

$$
\frac{\partial S}{\partial \ddot{\pi}}=\Pi_{\mathfrak{F}}+\Pi_{\mathfrak{C}}
$$

where $S$ is the energy of accelerations of the system, $\ddot{\pi}$ is the column of pseudo-accelerations, $\boldsymbol{\Pi}_{\mathfrak{C}}$ is the column of generalized control forces reduced to pseudo-velocities, and $\boldsymbol{\Pi}_{\mathfrak{F}}$ is the column of other generalized forces. Here

$$
\ddot{\pi}=\left(\begin{array}{lll}
\dot{V}_{X} & \dot{V}_{Y} & \dot{\Omega}
\end{array}\right)^{\mathrm{T}}, \quad \frac{\partial S}{\partial \ddot{\pi}}=\left(\begin{array}{lll}
\frac{\partial S}{\partial \dot{V}_{X}} & \frac{\partial S}{\partial \dot{V}_{Y}} & \frac{\partial S}{\partial \dot{\Omega}}
\end{array}\right)^{\mathrm{T}} .
$$

In the problem considered

$$
S=S_{p}+\sum_{i=1}^{4} S_{i}
$$

where $S_{p}$ are the energies of accelerations of the platform and of the wheel $i$, respectively.

The energy of accelerations of the platform is therefore $[6,14]$ :

$$
S_{p}=\frac{1}{2}\left\{m\left(w_{C X}^{2}+w_{C Y}^{2}\right)+I_{C} \dot{\Omega}^{2}\right\}
$$


where $w_{C X}=\dot{V}_{X}-a_{Y} \dot{\Omega}-\Omega\left(V_{Y}+a_{X} \Omega\right)$ and $w_{C Y}=\dot{V}_{Y}+a_{X} \dot{\Omega}+\Omega\left(V_{X}-a_{Y} \Omega\right)$ are the components of the acceleration of the platform's center of mass $C, m$ is the mass of the platform, $I_{C}$ is its inertia moment relative to the axis $C Z$, and $a_{X}$ and $a_{Y}$ are the coordinates of the center of mass $C$ in the plane $O X Y$. Here and in the sequel, in the expressions for energies of accelerations, we omit terms independent of pseudo-acceleration.

Differentiating $S_{p}$ with respect to pseudo-accelerations, we obtain

$$
\frac{\partial S_{p}}{\partial \ddot{\pi}}=\left(\begin{array}{c}
m\left(\dot{V}_{X}-\Omega V_{Y}\right)-m a_{Y} \dot{\Omega}-m a_{X} \Omega^{2} \\
m\left(\dot{V}_{Y}+\Omega V_{X}\right)+m a_{X} \dot{\Omega}-m a_{Y} \Omega^{2} \\
I_{O} \dot{\Omega}-m a_{Y}\left(\dot{V}_{X}-\Omega V_{Y}\right)+m a_{X}\left(\dot{V}_{Y}+\Omega V_{X}\right)
\end{array}\right),
$$

where $I_{O}=I_{C}+m\left(a_{X}^{2}+a_{Y}^{2}\right)$ is the moment of inertia of the platform relative to the axis $O Z$.

Assuming that the center of mass of the $i$ th wheel coincides with the geometric center $C_{i}$ and $C_{i} Y$ is the axis of dynamical symmetry, the expression for $S_{i}$ takes the form $[6,14,36]$ :

$$
S_{i}=\frac{1}{2}\left\{m_{1} \boldsymbol{w}_{C_{i}}^{2}+I_{1} \dot{\Omega}^{2}+J_{1} \ddot{\varphi}_{i}^{2}\right\},
$$

where $m_{1}$ is the mass of the wheel, $J_{1}$ and $I_{1}$ are its inertia moments relative to the axes $C_{i} Y$ and $C_{i} Z$, respectively, and $\boldsymbol{w}_{C_{i}}$ is the acceleration of the center of mass $C_{i}$.

The total energy of accelerations of the four-wheel system is

$$
\begin{aligned}
\sum S_{i}= & \frac{1}{2}\left\{4 I_{1} \dot{\Omega}^{2}+J_{1} \ddot{\boldsymbol{\varphi}}^{\mathrm{T}} \ddot{\boldsymbol{\varphi}}+m_{1} \sum \boldsymbol{w}_{C_{i}}^{2}\right\}= \\
= & \frac{1}{2}\left\{4 I_{1} \dot{\Omega}^{2}+J_{1} \ddot{\boldsymbol{\pi}}^{\mathrm{T}} \boldsymbol{C}_{\varphi}^{\mathrm{T}} \boldsymbol{C}_{\varphi} \ddot{\boldsymbol{\pi}}+4 m_{1}\left(w_{O X}^{2}+w_{O Y}^{2}\right)+4 m_{1}\left(l^{2}+h^{2}\right) \dot{\Omega}^{2}\right\}= \\
& =2\left\{\left(I_{1}+m_{1}\left(l^{2}+h^{2}\right)\right) \dot{\Omega}^{2}+\frac{J_{1}}{R^{2}}\left(\dot{V}_{X}^{2}+\dot{V}_{Y}^{2}+(l+h)^{2} \dot{\Omega}^{2}\right)+m_{1}\left(w_{O X}^{2}+w_{O Y}^{2}\right)\right\},
\end{aligned}
$$

where $w_{O X}=\dot{V}_{X}-\Omega V_{Y}$ and $w_{O Y}=\dot{V}_{Y}+\Omega V_{X}$ are the acceleration components of the point $O$, which is the center of mass of the wheel system, and the matrix $C_{\varphi}^{\mathrm{T}} \boldsymbol{C}_{\varphi}=\frac{4}{R^{2}} \operatorname{diag}\left(1,1,(l+h)^{2}\right)$.

The contribution of the wheels' inertia to the left-hand side of the Appel equations (2.5) is

$$
\sum_{i=1}^{4} \frac{\partial S_{i}}{\partial \ddot{\pi}}=\frac{4}{R^{2}}\left(\begin{array}{c}
m_{1} R^{2}\left(\dot{V}_{X}-\Omega V_{Y}\right)+J_{1} \dot{V}_{X} \\
m_{1} R^{2}\left(\dot{V}_{Y}+\Omega V_{X}\right)+J_{1} \dot{V}_{Y} \\
\left(I_{1}+m_{1}\left(h^{2}+l^{2}\right)\right) R^{2} \dot{\Omega}+J_{1}(l+h)^{2} \dot{\Omega}
\end{array}\right)
$$

In the problem considered the control torques of the actuators $M_{i}$, linear viscous friction torques in the wheel power transmission bearings, and also linear viscous friction torques in the bearings of the rollers in contact with the underlying surface are active force factors.

Let us draw the vector of generalized forces $\boldsymbol{\Pi}_{\mathfrak{F}}$ after computation of the work $\delta A_{\mathfrak{F}}$ of the viscous friction torques in the joints of the bodies, which is done in moving through the virtual displacements $\delta \boldsymbol{\varphi}=\boldsymbol{C}_{\varphi} \delta \boldsymbol{\pi}$ and $\delta \boldsymbol{\gamma}=\boldsymbol{C}_{\gamma} \delta \boldsymbol{\pi}$ :

$$
\delta A_{\mathfrak{F}}=\delta \boldsymbol{\varphi}^{\mathrm{T}}\left(-\mu_{1} \dot{\boldsymbol{\varphi}}\right)+\delta \boldsymbol{\gamma}^{\mathrm{T}}\left(-\mu_{2} \dot{\gamma}\right)=\delta \boldsymbol{\pi}^{\mathrm{T}}\left(-\mu_{1} \boldsymbol{C}_{\varphi}^{\mathrm{T}} \boldsymbol{C}_{\boldsymbol{\varphi}}-\mu_{1} \boldsymbol{C}_{\gamma}^{\mathrm{T}} \boldsymbol{C}_{\gamma}\right) \dot{\boldsymbol{\pi}}
$$


Here $\delta \boldsymbol{\pi}$ is the vector of variations with independent components; $\mu_{1}$ is the coefficient of viscous friction in the joints of the wheels and of the platform, and $\mu_{2}$ is the coefficient of viscous friction in the bearings of the rollers.

Since $\delta A_{\mathfrak{F}}=\delta \boldsymbol{\pi}^{\mathrm{T}} \boldsymbol{\Pi}_{\mathfrak{F}}$, we have

$$
\boldsymbol{\Pi}_{\mathfrak{F}}=-\left(\mu_{1} \boldsymbol{C}_{\varphi}^{\mathrm{T}} \boldsymbol{C}_{\varphi}+\mu_{1} \boldsymbol{C}_{\gamma}^{\mathrm{T}} \boldsymbol{C}_{\gamma}\right) \dot{\boldsymbol{\pi}}=-\left(\begin{array}{lll}
\zeta_{X} V_{X} & \zeta_{Y} V_{Y} & \mu_{\Omega} \Omega
\end{array}\right)^{\mathrm{T}},
$$

where $\zeta_{X}, \zeta_{Y}$ and $\mu_{\Omega}$ are the reduced coefficients of damping:

$$
\zeta_{X}=\frac{4 \mu_{1}}{R^{2}}, \quad \zeta_{Y}=\frac{4 \mu_{1}}{R^{2}}+\frac{8 \mu_{2}}{r^{2}}, \quad \mu_{\Omega}=\frac{4 \mu_{1}(l+h)^{2}}{R^{2}}+\frac{8 \mu_{2} h^{2}}{r^{2}} .
$$

We note that taking into account linear friction forces on the axes of the rollers results in the appearance of anisotropic generalized forces of viscous friction in the equations of motion.

Remark. The position of the contact point on the roller surface, the radius of its cross section touching the floor, and the orientation of the roller axis depend on the rotation angle of the Mecanum wheel. In this connection, we observe that the coefficient $\mu_{2} / r^{2}$ in the last formulae defines the "averaged" dependence of generalized forces on viscous friction in the supports of the rollers.

Calculating the virtual work

$$
\delta A_{\mathfrak{C}}=\delta \boldsymbol{\varphi}^{\mathrm{T}} \boldsymbol{M}=\delta \boldsymbol{\pi}^{\mathrm{T}} \boldsymbol{C}_{\varphi}^{\mathrm{T}} \boldsymbol{M}=\delta \boldsymbol{\pi}^{\mathrm{T}} \boldsymbol{\Pi}_{\mathfrak{C}}
$$

of the control torques forming the column $\boldsymbol{M}=\left(\begin{array}{llll}M_{1} & M_{2} & M_{3} & M_{4}\end{array}\right)^{\mathrm{T}}$, we find the vector of the generalized control forces

$$
\boldsymbol{\Pi}_{\mathfrak{C}}=\boldsymbol{C}_{\varphi}^{\mathrm{T}} \boldsymbol{M}=\frac{1}{R}\left(\begin{array}{c}
M_{2}+M_{1}+M_{4}+M_{3} \\
M_{2}-M_{1}-M_{4}+M_{3} \\
(l+h)\left[M_{2}-M_{1}+M_{4}-M_{3}\right]
\end{array}\right) \equiv\left(\begin{array}{c}
F_{X} \\
F_{Y} \\
M_{\Omega}
\end{array}\right) .
$$

Adding the left-hand and right-hand sides of the Appel equation (2.5) using (2.8) and (2.10) and the expressions for the generalized forces (2.11) and (2.12), we obtain

$$
\begin{gathered}
m_{a} \dot{V}_{X}-m_{s} \Omega V_{Y}-m a_{Y} \dot{\Omega}-m a_{X} \Omega^{2}+\zeta_{X} V_{X}=F_{X}, \\
m_{a} \dot{V}_{Y}+m_{s} \Omega V_{X}+m a_{X} \dot{\Omega}-m a_{Y} \Omega^{2}+\zeta_{Y} V_{Y}=F_{Y}, \\
I_{a} \dot{\Omega}-m a_{Y}\left(\dot{V}_{X}-\Omega V_{Y}\right)+m a_{X}\left(\dot{V}_{Y}+\Omega V_{X}\right)+\mu_{\Omega} \Omega=M_{\Omega},
\end{gathered}
$$

where $m_{s}=m+4 m_{1}$ is the total mass of the robot, $I_{s}=I_{O}+4\left(I_{1}+m_{1}\left(h^{2}+l^{2}\right)\right)$ is its moment of inertia relative to the axis $O Z$, and $m_{a}=m_{s}+\frac{4}{R^{2}} J_{1}$ and $I_{a}=I_{s}+\frac{4(l+h)^{2}}{R^{2}} J_{1}$ are the reduced mass and the reduced moment of inertia, respectively.

The vector-matrix form of representation of Eqs. (2.13)-(2.15) is

$$
A \ddot{\pi}+f-\Pi_{\mathfrak{F}}=\Pi_{\mathfrak{C}}
$$


where the matrix $\boldsymbol{A}$ is the matrix of inertia coefficients, $\boldsymbol{f}$ is the column of quadratic forms from pseudo-velocities:

$$
\boldsymbol{A}=\left(\begin{array}{ccc}
m_{a} & 0 & -m a_{Y} \\
0 & m_{a} & m a_{X} \\
-m a_{Y} & m a_{X} & I_{a}
\end{array}\right), \quad \boldsymbol{f}=\left(\begin{array}{c}
-m_{s} \Omega V_{Y}-m a_{X} \Omega^{2} \\
m_{s} \Omega V_{X}-m a_{Y} \Omega^{2} \\
m a_{Y} \Omega V_{Y}+m a_{X} \Omega V_{X}
\end{array}\right)
$$

Further, in investigating the dynamics of the system, we will use the following parameter values $[1,37]$ :

$$
\begin{gathered}
m=29.94 \mathrm{~kg}, \quad m_{1}=1.40 \mathrm{~kg}, \quad I_{s}=4.42 \mathrm{~kg} \cdot \mathrm{m}^{2}, \\
J_{1}=5.87 \cdot 10^{-3} \mathrm{~kg} \cdot \mathrm{m}^{2}, \quad a_{X}=1.6 \mathrm{~cm}, \quad a_{Y}=-2.6 \mathrm{~cm}, \\
\mu_{1}=0.110 \mathrm{~N} \cdot \mathrm{m} \cdot \mathrm{s}, \quad \mu_{2}=5.78 \cdot 10^{-3} \mathrm{~N} \cdot \mathrm{m} \cdot \mathrm{s}, \\
l=0.150 \mathrm{~m}, \quad h=0.235 \mathrm{~m}, \quad R=0.0475 \mathrm{~m}, \quad r=0.014 \mathrm{~m} .
\end{gathered}
$$

\section{The use of nonholonomic constraints in the problem of controlling the motion of the platform}

The problem is to implement the program motion of the platform with a given velocity vector of some point $B$ of the platform:

$$
V_{B X}=V_{B X}^{*}, \quad V_{B Y}=V_{B Y}^{*},
$$

where $V_{B X}, V_{B Y}, V_{B X}^{*}$, and $V_{B Y}^{*}$ are the projections of the velocity vector, and their laws of variation during the program motion, respectively. In the general case, the quantities $V_{B X}^{*}$ and $V_{B Y}^{*}$ explicitly depend not only on time, but also on the vector of generalized coordinates $\boldsymbol{q}$, on measurements of the coordinates of the bodies with respect to which the platform must maneuver, and on other quantities.

Let us write Eq. (3.1) by expressing $V_{B X}$ and $V_{B Y}$ in terms of pseudo-velocities $V_{X}, V_{Y}$ and $\Omega$ :

$$
V_{X}-b_{Y} \Omega=V_{B X}^{*}, \quad V_{Y}+b_{X} \Omega=V_{B Y}^{*},
$$

where $b_{X}$ and $b_{Y}$ are the abscissa and the ordinate of the point $B$ in the plane $O X Y$. We rewrite these relations in the vector-matrix form:

$$
\boldsymbol{B} \dot{\boldsymbol{\pi}}=\boldsymbol{V}_{B}^{*}, \quad \boldsymbol{B}=\left(\begin{array}{ccc}
1 & 0 & -b_{Y} \\
0 & 1 & b_{X}
\end{array}\right), \quad \boldsymbol{V}_{B}^{*}=\left(\begin{array}{c}
V_{B X}^{*} \\
V_{B Y}^{*}
\end{array}\right) .
$$

We now present the general algorithm for finding control forces to implement the required motion (3.2).

Three generalized control forces on the program motion cannot be uniquely found from the equations of motion, since the number of additional relations (3.2) is less than the number of degrees of freedom of the system under study. 
Let us consider relations (3.2) as additional ideal constraints imposed on the robot dynamics. The equations for the uncontrollable motion of the system with such constraints take the form $[2,17]$ :

$$
\frac{\partial S}{\partial \ddot{\boldsymbol{\pi}}}=\boldsymbol{\Pi}_{\mathfrak{F}}+\boldsymbol{B}^{\mathrm{T}} \boldsymbol{\lambda}
$$

where $\boldsymbol{\lambda}=\left(\begin{array}{ll}\lambda_{1} & \lambda_{2}\end{array}\right)^{\mathrm{T}}$ is the vector of the multipliers of additional constraints.

Solving the system (3.2)-(3.4), which, if necessary, is closed with kinematics equations, we find the law of variation of $\boldsymbol{\lambda}$ for the program motion and, identifying the reactions of additional constraints (3.2) with the control forces, we find the vector $\boldsymbol{\Pi}_{\mathfrak{C}}$ :

$$
\Pi_{\mathfrak{C}}=\boldsymbol{B}^{\mathrm{T}} \boldsymbol{\lambda}
$$

We eliminate the multipliers of constraints from the equations of motion. Next, we express the longitudinal and transverse velocity components of the platform's center in terms of $\Omega$ :

$$
V_{X}=b_{Y} \Omega+V_{B X}^{*}, \quad V_{Y}=-b_{X} \Omega+V_{B Y}^{*},
$$

which may be represented as

$$
\dot{\boldsymbol{\pi}}=\boldsymbol{C} \Omega+\boldsymbol{V}^{*}, \quad \boldsymbol{C}=\left(\begin{array}{c}
b_{Y} \\
-b_{X} \\
1
\end{array}\right), \quad \boldsymbol{V}^{*}=\left(\begin{array}{c}
V_{B X}^{*} \\
V_{B Y}^{*} \\
0
\end{array}\right) .
$$

It is easy to check that the product of matrices $\boldsymbol{B C}=0$.

Thus, multiplying both sides of the system (3.4) by $\boldsymbol{C}^{\mathrm{T}}$, we obtain an equation that does not contain the multipliers of constraints

$$
\boldsymbol{C}^{\mathrm{T}} \frac{\partial S}{\partial \ddot{\pi}}=\boldsymbol{C}^{\mathrm{T}} \boldsymbol{\Pi}_{\mathfrak{F}}
$$

In the equality obtained we express the pseudo-velocities $V_{X}$ and $V_{Y}$ in terms of $\Omega$ using the matrix relations (2.16) and (3.7). We thus obtain the scalar differential equation

$$
\boldsymbol{C}^{\mathrm{T}} \boldsymbol{A} \boldsymbol{C} \dot{\Omega}+\boldsymbol{C}^{\mathrm{T}} \boldsymbol{A} \dot{\boldsymbol{V}}^{*}+\boldsymbol{C}^{\mathrm{T}} \boldsymbol{f}=\boldsymbol{C}^{\mathrm{T}} \boldsymbol{\Pi}_{\mathfrak{F}}
$$

To obtain the explicit form of this relation, we perform all necessary matrix operations. We have

$$
\boldsymbol{A C}=\left(\begin{array}{c}
m_{a} b_{Y}-m a_{Y} \\
-m_{a} b_{X}+m a_{X} \\
I_{a}-m a_{Y} b_{Y}-m a_{X} b_{X}
\end{array}\right) .
$$

We write the expression for the inertia coefficient $J_{a}$ in Eq. (3.8):

$$
\begin{aligned}
J_{a}= & \boldsymbol{C}^{\mathrm{T}} \boldsymbol{A} \boldsymbol{C}=I_{a}-2 m\left(a_{X} b_{X}+a_{Y} b_{Y}\right)+m_{a}\left(b_{X}^{2}+b_{Y}^{2}\right)= \\
& =I_{C}+m\left(\left[a_{X}-b_{X}\right]^{2}+\left[a_{Y}-b_{Y}\right]^{2}\right)+4\left[I_{1}+m_{1}\left(h^{2}+l^{2}+b_{X}^{2}+b_{Y}^{2}\right)+J_{1} \frac{(l+h)^{2}+b_{X}^{2}+b_{Y}^{2}}{R^{2}}\right] .
\end{aligned}
$$


Let us find expressions for other quantities in the formula (3.8) by expressing the velocities $V_{X}$ and $V_{Y}$ in terms of the independent pseudo-velocity $\Omega$ in accordance with (3.6):

$$
\begin{gathered}
\boldsymbol{C}^{\mathrm{T}} \boldsymbol{A} \dot{\boldsymbol{V}}^{*}=\left(m_{a} b_{Y}-m a_{Y}\right) \dot{V}_{B X}^{*}-\left(m_{a} b_{X}-m a_{X}\right) \dot{V}_{B Y}^{*} ; \\
\boldsymbol{C}^{\mathrm{T}} \boldsymbol{f}=-\left(m_{s} b_{X}-m a_{X}\right) \Omega V_{B X}^{*}-\left(m_{s} b_{Y}-m a_{Y}\right) \Omega V_{B Y}^{*} ; \\
\boldsymbol{C}^{\mathrm{T}} \boldsymbol{\Pi}_{\mathfrak{F}}=-\zeta_{X} b_{Y} V_{B X}^{*}+\zeta_{Y} b_{X} V_{B Y}^{*}-\left(\mu_{\Omega}+\zeta_{X} b_{Y}^{2}+\zeta_{Y} b_{X}^{2}\right) \Omega .
\end{gathered}
$$

We substitute the results obtained into Eq. (3.8):

$$
\begin{aligned}
J_{a} \dot{\Omega}+\left(m_{a} b_{Y}-m a_{Y}\right) \dot{V}_{B X}^{*}-\left(m_{a} b_{X}-m a_{X}\right) \dot{V}_{B Y}^{*}- & \\
-\left(m_{s} b_{X}-m a_{X}\right) \Omega V_{B X}^{*}-\left(m_{s} b_{Y}\right. & \left.-m a_{Y}\right) \Omega V_{B Y}^{*}+\zeta_{X} b_{Y} V_{B X}^{*}- \\
& -\zeta_{Y} b_{X} V_{B Y}^{*}+\left(\mu_{\Omega}+\zeta_{X} b_{Y}^{2}+\zeta_{Y} b_{X}^{2}\right) \Omega=0 .
\end{aligned}
$$

Dividing both sides of this equality by $J_{a}$, we obtain an equation that is satisfied by the angular velocity of the platform $\Omega$ during the program motion:

$$
\dot{\Omega}+\varkappa_{12} \dot{V}_{B X}^{*}-\varkappa_{11} \dot{V}_{B Y}^{*}-\varkappa_{21} \Omega V_{B X}^{*}-\varkappa_{22} \Omega V_{B Y}^{*}+\xi_{2} V_{B X}^{*}-\xi_{1} V_{B Y}^{*}+\xi_{3} \Omega=0,
$$

where

$$
\begin{gathered}
\varkappa_{11}=\frac{m_{a} b_{X}-m a_{X}}{J_{a}}, \quad \varkappa_{12}=\frac{m_{a} b_{Y}-m a_{Y}}{J_{a}}, \\
\varkappa_{21}=\frac{m_{s} b_{X}-m a_{X}}{J_{a}}, \quad \varkappa_{22}=\frac{m_{s} b_{Y}-m a_{Y}}{J_{a}}, \\
\xi_{1}=\frac{\zeta_{Y} b_{X}}{J_{a}}, \quad \xi_{2}=\frac{\zeta_{X} b_{Y}}{J_{a}}, \quad \xi_{3}=\frac{\mu_{\Omega}+\zeta_{X} b_{Y}^{2}+\zeta_{Y} b_{X}^{2}}{J_{a}} .
\end{gathered}
$$

Solving Eq. (3.9), we get the angular velocity $\Omega$. Using $\Omega$, we find $V_{X}$ and $V_{Y}$ by formulae (3.6).

The composition of the matrix $\boldsymbol{B}$ of the constraint equations (3.2) allows us to obtain expressions for the multipliers of constraints for the program motion by substituting the obtained values of $\Omega, V_{X}$ and $V_{Y}$ into the first two equations of the system (3.4):

$$
\begin{gathered}
\lambda_{1}=m_{a} \dot{V}_{X}-m a_{Y} \dot{\Omega}-m_{s} \Omega V_{Y}-m a_{X} \Omega^{2}+\zeta_{X} V_{X}, \\
\lambda_{2}=m_{a} \dot{V}_{Y}+m a_{X} \dot{\Omega}+m_{s} \Omega \lambda_{2}=m_{a} \dot{V}_{Y}+m a_{X} \dot{\Omega}+m_{s} \Omega V_{X}-m a_{Y} \Omega^{2}+\zeta_{Y} V_{Y} .
\end{gathered}
$$

According to (3.5), the generalized control forces involved in the program motion are computed as follows:

$$
F_{X}=\lambda_{1}, \quad F_{Y}=\lambda_{2}, \quad M_{\Omega}=-b_{Y} \lambda_{1}+b_{X} \lambda_{2} .
$$

We note that the quantities of the four control torques $M_{i}(i=\overline{1,4})$ are determined nonuniquely from the known values of the three generalized forces $F_{X}, F_{Y}$ and $M_{\Omega}$. To calculate the moments of the actuators, we choose a solution with the minimal Euclidean norm [5, p. 34], [33] from an uncountable set of solutions of the system $\boldsymbol{C}_{\varphi}^{\mathrm{T}} \boldsymbol{M}=\boldsymbol{\Pi}_{\mathfrak{C}}$ :

$$
\begin{array}{cc}
\boldsymbol{M}=\boldsymbol{C}_{\varphi}\left(\boldsymbol{C}_{\varphi}^{\mathrm{T}} \boldsymbol{C}_{\varphi}\right)^{-1} \boldsymbol{\Pi}_{\mathfrak{C}} ; & \left.M_{\Omega}\right\}, \\
M_{1}=\frac{R}{4}\left\{F_{X}-F_{Y}-\frac{M_{\Omega}}{h+l}\right\}, & M_{2}=\frac{R}{4}\left\{F_{X}+F_{Y}+\frac{M_{\Omega}}{h+l}\right\}, \\
M_{3}=\frac{R}{4}\left\{F_{X}+F_{Y}-\frac{M_{\Omega}}{h+l}\right\}, & M_{4}=\frac{R}{4}\left\{F_{X}-F_{Y}+\frac{M_{\Omega}}{h+l}\right\} .
\end{array}
$$




\section{The problem of implementation of the motion of the platform in a circle}

Let us proceed to specific examples of application of the algorithm developed to control the motion of the youBot platform.

Let us specify the uniform motion of point $B$ in a circle of radius $R_{B}$ with angular frequency $\omega_{B} \neq 0$ as program motion. The laws of variation of the coordinates of the point $B$ on the fixed plane $x y$ have the following form:

$$
x_{B}^{*}=R_{B} \cos \left(\omega_{B} t+\varphi_{B}\right), \quad y_{B}^{*}=R_{B} \sin \left(\omega_{B} t+\varphi_{B}\right),
$$

where $\varphi_{B}=$ const is the initial phase.

For such program motion, the projections of the velocity of point $B$ onto the moving axes $O X Y$ are

$$
\begin{aligned}
& V_{B X}^{*}=\dot{x}_{B}^{*} \cos \psi+\dot{y}_{B}^{*} \sin \psi=R_{B} \omega_{B} \sin \left(\psi-\omega_{B} t-\varphi_{B}\right), \\
& V_{B Y}^{*}=-\dot{x}_{B}^{*} \sin \psi+\dot{y}_{B}^{*} \cos \psi=R_{B} \omega_{B} \cos \left(\psi-\omega_{B} t-\varphi_{B}\right) .
\end{aligned}
$$

Denote $\widetilde{\psi}=\psi-\omega_{B} t-\varphi_{B}$. Then

$$
\Omega=\dot{\widetilde{\psi}}+\omega_{B}, \quad V_{B X}^{*}=R_{B} \omega_{B} \sin \widetilde{\psi}, \quad V_{B Y}^{*}=R_{B} \omega_{B} \cos \tilde{\psi} .
$$

We now investigate the angular motion of the platform of the youBot platform with the program motion (4.2) compatible with the additional constraints.

Substituting the expressions (4.2) into Eq. (3.9) for the angular velocity of the platform, $\Omega$, during the motion with the constraints (3.2), (4.1), we obtain

$$
\begin{aligned}
\ddot{\widetilde{\psi}}+\xi_{3}\left(\dot{\widetilde{\psi}}+\omega_{B}\right)+R_{B} \omega_{B}\left[\varkappa_{12} \dot{\widetilde{\psi}}-\xi_{1}\right. & \left.-\varkappa_{22}\left(\dot{\widetilde{\psi}}+\omega_{B}\right)\right] \cos \widetilde{\psi}+ \\
& +R_{B} \omega_{B}\left[\varkappa_{11} \dot{\widetilde{\psi}}+\xi_{2}-\varkappa_{21}\left(\dot{\widetilde{\psi}}+\omega_{B}\right)\right] \sin \widetilde{\psi}=0 .
\end{aligned}
$$

Let us rewrite the last equation as

$$
\ddot{\widetilde{\psi}}+\xi_{3}\left\{1+\frac{R_{B} \omega_{B}}{\widetilde{V}_{B}} \sin (\widetilde{\psi}+\beta)\right\} \dot{\widetilde{\psi}}+\omega_{B} \xi_{3}\left\{1-\frac{R_{B}}{\widetilde{R}_{B}} \cos (\widetilde{\psi}+\alpha)\right\}=0,
$$

where

$$
\begin{gathered}
\widetilde{R}_{B}=\frac{\xi_{3}}{\sqrt{\left(\xi_{1}+\varkappa_{22} \omega_{B}\right)^{2}+\left(\xi_{2}-\varkappa_{21} \omega_{B}\right)^{2}}}, \\
\widetilde{V}_{B}=\frac{\xi_{3}}{\sqrt{\left(\varkappa_{11}-\varkappa_{21}\right)^{2}+\left(\varkappa_{12}-\varkappa_{22}\right)^{2}}}, \\
\cos \alpha=\left(\xi_{1}+\varkappa_{22} \omega_{B}\right) \frac{\widetilde{R}_{B}}{\xi_{3}}, \quad \sin \alpha=\left(\xi_{2}-\varkappa_{21} \omega_{B}\right) \frac{\widetilde{R}_{B}}{\xi_{3}}, \\
\cos \beta=\left(\varkappa_{11}-\varkappa_{21}\right) \frac{\widetilde{V}_{B}}{\xi_{3}}, \quad \sin \beta=\left(\varkappa_{12}-\varkappa_{22}\right) \frac{\widetilde{V}_{B}}{\xi_{3}} .
\end{gathered}
$$

Let us examine the properties of the solutions to Eq. (4.4) depending on the parameters of the program motion $\omega_{B}$ and $R_{B}$. 


\subsection{Steady rotations of the platform}

The motion of the platform, which is steady with respect to the angular velocity $\Omega=\omega_{B}$, is of special interest, since it is intuitively predictable in the problem considered and is simple to implement. Let us find conditions for the existence of such steady-state regimes and investigate their stability.

Consider the solution to Eq. (4.4) $\widetilde{\psi}=\widetilde{\psi}_{s}=$ const. Substituting it into (4.4), we obtain

$$
\omega_{B} \xi_{3}\left\{1-\frac{R_{B}}{\widetilde{R}_{B}} \cos \left(\widetilde{\psi}_{s}+\alpha\right)\right\}=0 .
$$

Analysis of the resulting equation shows that the constant $\widetilde{\psi}_{s}$ characterizing the platform's motion regime, which is steady with respect to the angular velocity, satisfies the relation

$$
\cos \left(\widetilde{\psi}_{s}+\alpha\right)=\frac{\widetilde{R}_{B}}{R_{B}}
$$

If $R_{B} \geqslant \widetilde{R}_{B}$, then the roots of the last equation are real, and steady rotations exist.

For $R_{B}>\widetilde{R}_{B}$ the roots of Eq. (4.6) have the following form:

$$
\widetilde{\psi}_{s 1}[k]=\arccos \frac{\widetilde{R}_{B}}{R_{B}}-\alpha+2 \pi k, \quad \widetilde{\psi}_{s 2}[k]=-\arccos \frac{\widetilde{R}_{B}}{R_{B}}-\alpha+2 \pi k, \quad k \in \mathbb{Z} .
$$

Since $R_{B}>0$ and $\widetilde{R}_{B}>0$, it follows that

$$
-\frac{\pi}{2}<\widetilde{\psi}_{s 1}[0]+\alpha<0, \quad 0<\widetilde{\psi}_{s 2}[0]+\alpha<\frac{\pi}{2} .
$$

Note that for $R_{B}=\widetilde{R}_{B}$ the roots of Eq. (4.6) are

$$
\widetilde{\psi}_{s}[k]=-\alpha+2 \pi k, \quad k \in \mathbb{Z} .
$$

Thus, $\widetilde{R}_{B}$ is the critical value of the radius of the program trajectory $R_{B}$ from the viewpoint of the existence of steady motions.

Let us now explore the linear stability of steady rotations. We introduce the deviation in the angle of rotation of the platform

$$
\Delta \psi=\widetilde{\psi}-\widetilde{\psi}_{s}=\psi-\omega_{B} t-\varphi_{B}-\widetilde{\psi}_{s} .
$$

We linearize Eq. (4.4) in a neighborhood of the steady-state solution $\widetilde{\psi}=\widetilde{\psi}_{s}$ :

$$
\Delta \ddot{\psi}+\xi_{3}\left\{1+\frac{R_{B} \omega_{B}}{\widetilde{V}_{B}} \sin \left(\widetilde{\psi}_{s}+\beta\right)\right\} \Delta \dot{\psi}+\frac{R_{B}}{\widetilde{R}_{B}} \omega_{B} \xi_{3} \sin \left(\widetilde{\psi}_{s}+\alpha\right) \Delta \psi=0 .
$$

The condition for the asymptotic stability of steady rotations implies that the coefficients of this equation are simultaneously positive for $\Delta \psi$ and $\Delta \dot{\psi}$.

Let $R_{B}>\widetilde{R}_{B}$. Since $\sin \left(\widetilde{\psi}_{s 1}[k]+\alpha\right)<0$ and $\sin \left(\widetilde{\psi}_{s 2}[k]+\alpha\right)>0$, it follows that, when $\omega_{B}>0$, the coefficient at $\Delta \psi$ in (4.7) is negative and, therefore, the steady-state regimes with 
$\widetilde{\psi}_{s}=\widetilde{\psi}_{s 1}[k]$ are unstable. By analogy, the steady-state regimes of the platform motion with $\widetilde{\psi}_{s}=\widetilde{\psi}_{s 2}[k]$ are unstable when $\omega_{B}<0$.

Note that, when $R_{B}=\widetilde{R}_{B}$, the coefficient at $\Delta \psi$ in (4.7) vanishes. Thus, the analysis of the linearized equation (4.7) yields only sufficient conditions for instability of the steady-state regimes with $\widetilde{\psi}_{s}=\widetilde{\psi}_{s}[k]$.

A sufficient (but not necessary) condition for positiveness of the coefficient at $\Delta \dot{\psi}$ in the linearized equation (4.7) has the form $R_{B}\left|\omega_{B}\right|<\widetilde{V}_{B}$.

Thus, the system of inequalities

$$
R_{B}>\widetilde{R}_{B}, \quad R_{B}\left|\omega_{B}\right|<\widetilde{V}_{B}
$$

defines sufficient conditions for the existence of asymptotically stable steady motion of the platform with angular velocity $\Omega=\omega_{B}$. If the above two conditions are satisfied and $\omega_{B}>0$, then the family of steady rotations with $\widetilde{\psi}_{s}=\widetilde{\psi}_{s 2}[k]$ is asymptotically stable, and if $\omega_{B}<0$, the family of steady rotations with $\widetilde{\psi}_{s}=\widetilde{\psi}_{s 1}[k]$ is asymptotically stable.

Relations (4.8) specify the subset of the domain of asymptotic stability of the steady-state regimes under study. Since the calculations involved are rather lengthy, this domain was built by symbolic transformations in Mathematica system. The stability domain of the steady-state regimes in the plane $\left(\omega_{B}, R_{B}\right)$ is presented in Fig. 3. To build the domain, the values $b_{X}=$ $=40 \mathrm{~cm}$ and $b_{Y}=5 \mathrm{~cm}$ and the numerical parameter values of the mathematical model of the robot (2.17) were used. The above values will be used in the sequel.

Figure 3 illustrates that for the selected parameter values all steady motions of the class under study are unstable for such large absolute values of $\omega_{B}$ that the motion (4.1) cannot be executed by the mobile youBot platform in practice. We also note that the stability domain built by using the sufficient condition (4.8) is essentially narrowed. However, it is justified to use conditions (4.8) in applications, since, for the chosen values of $b_{X}$ and $b_{Y}$, the "critical" velocity $\widetilde{V}_{B}=26.58 \mathrm{~m} / \mathrm{s}$ is many times greater than the maximal platform velocity $0.8 \mathrm{~m} / \mathrm{s}[37]$.

We note that if the moment of inertia of each wheel is $J_{1}=0$, then in the equation of the angular motion of the platform in the deviations (4.7) the coefficient at $\Delta \dot{\psi}$ will be positive and equal to $\xi_{3}$. Indeed, when $J_{1}=0$, the values of $m_{a}$ and $m_{s}$ will be the same and, by the formulae $(3.10), \varkappa_{11}-\varkappa_{21}=\varkappa_{12}-\varkappa_{22}=0$.

Indeed, for zero value of $J_{1}$ one can always find asymptotically stable motions among the motions with $\Omega(t) \equiv \omega_{B}$. The inertia of the wheels has a destabilizing effect on the steady-state regimes with respect to the angular velocity of the platform: all the regimes become unstable due to negativeness of the coefficient at $\Delta \dot{\psi}$ in Eq. (4.7) for some combinations of the parameter values.

The results of the mathematical modeling for the motion of the mobile youBot platform are as follows. The program motion (4.1) is characterized by the parameters of $\omega_{B}=-0.4 \mathrm{~s}^{-1}$, $R_{B}=0.7 \mathrm{~m}, \varphi_{B}=90^{\circ}$. The abscissa and the ordinate of $B$ in the plane $O X Y$ are: $b_{X}=0.4 \mathrm{~m}$, $b_{Y}=0.05 \mathrm{~m}$. At the initial time $\psi(0)=0, \Omega(0)=0$.

The angular velocity of the platform obeys Eq. (4.4), and the generalized control forces are computed by the formulae (3.11)-(3.13).

For the parameter values used in modeling, $\widetilde{R}_{B}=0.65 \mathrm{~m}$ and $\widetilde{V}_{B}=26.58 \mathrm{~m} / \mathrm{s}$. Thus, in the problem under consideration, the sufficient conditions (4.8) for the existence of stable steady (with respect to the angular velocity) motion of the platform where $\widetilde{\psi}_{s}=-0.48$ are satisfied. This is evidenced by the results of numerical simulation of the controlled motion, 


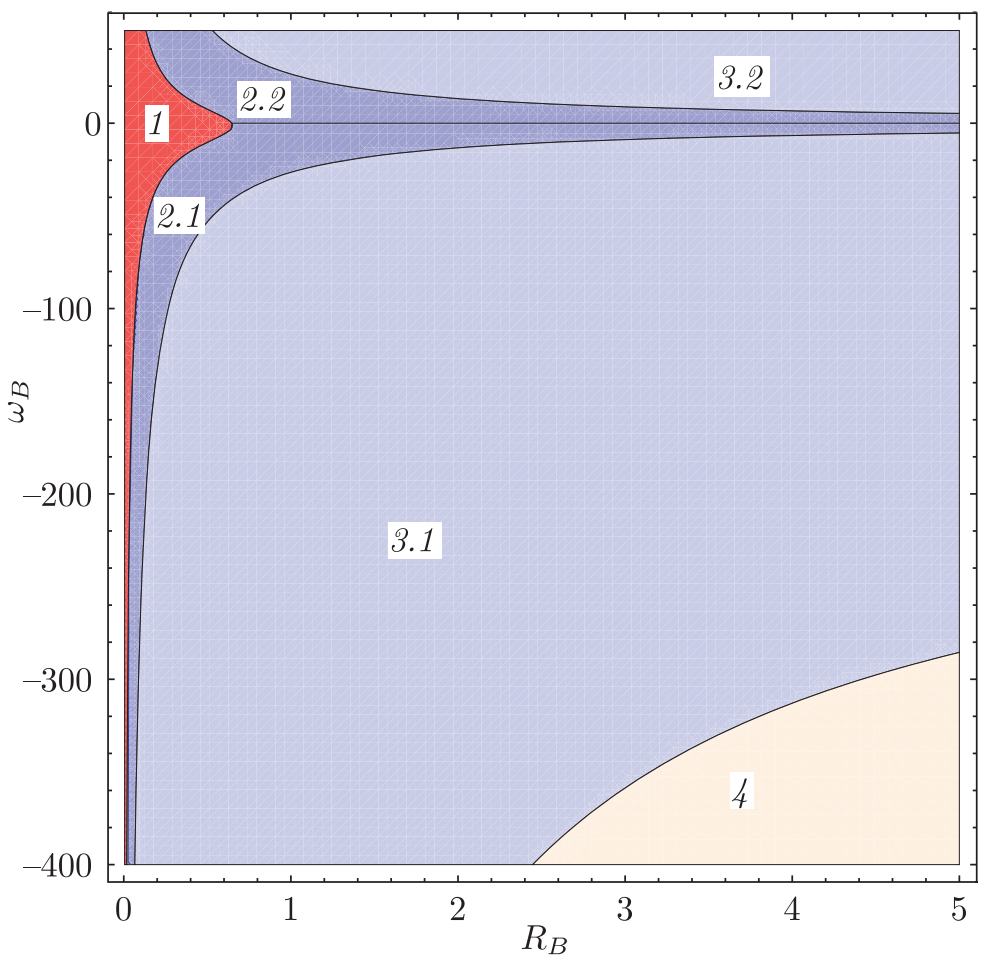

Steady motions with $\Omega=\omega_{B}: 1$ do not exist;

2.1 and 3.1 are stable for $\widetilde{\psi}_{s}=\widetilde{\psi}_{s 1}[k]$, and are unstable for $\widetilde{\psi}_{s}=\widetilde{\psi}_{s 2}[k]$;

2.1 are stable for $\widetilde{\psi}_{s}=\widetilde{\psi}_{s 1}[k]$ in accordance with (4.8);

2.2 and 3.2 are stable for $\widetilde{\psi}_{s}=\widetilde{\psi}_{s 2}[k]$, and are unstable for $\widetilde{\psi}_{s}=\widetilde{\psi}_{s 1}[k]$;

2.2 are stable for $\widetilde{\psi}_{s}=\widetilde{\psi}_{s 2}[k]$ in accordance with (4.8);

4 are all unstable.

Fig. 3. Domains of existence, asymptotic stability and instability of the controlled motions of the mobile youBot platform which are steady with respect to the angular velocity.

namely, the trajectories of the points of the robot's platform and the dependence $\Delta \psi(t)=$ $=\psi(t)-\omega_{B} t-\varphi_{B}-\widetilde{\psi}_{s}$, shown in Fig. 4 .

\subsection{Asymptotic Investigation of the Platform Motion}

Let us investigate the motion of the platform in a circle, assuming the viscous friction coefficients to be sufficiently large. This is confirmed by the values of the physical parameters of the system (2.17). Let us introduce the small parameter

$$
\varepsilon=\frac{\left|\omega_{B}\right|}{\xi_{3}} \ll 1
$$

Further, we introduce the nondimensional time $\tau=\left|\omega_{B}\right| t$ and denote $s_{\omega}=\operatorname{sign} \omega_{B}$. The equation of the angular motion takes the form

$$
\omega_{B}^{2} \frac{d^{2} \widetilde{\psi}}{d \tau^{2}}+\xi_{3}\left|\omega_{B}\right|\left\{1+\frac{R_{B} \omega_{B}}{\widetilde{V}_{B}} \sin (\widetilde{\psi}+\beta)\right\} \frac{d \widetilde{\psi}}{d \tau}+\omega_{B} \xi_{3}\left\{1-\frac{R_{B}}{\widetilde{R}_{B}} \cos (\widetilde{\psi}+\alpha)\right\}=0
$$




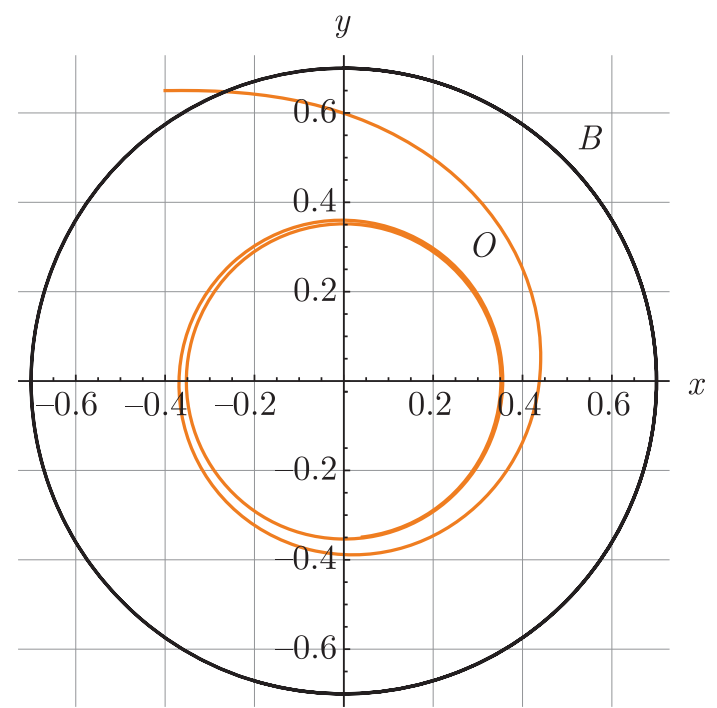

(a) Trajectories of point $B$ and the center of the platform $O$

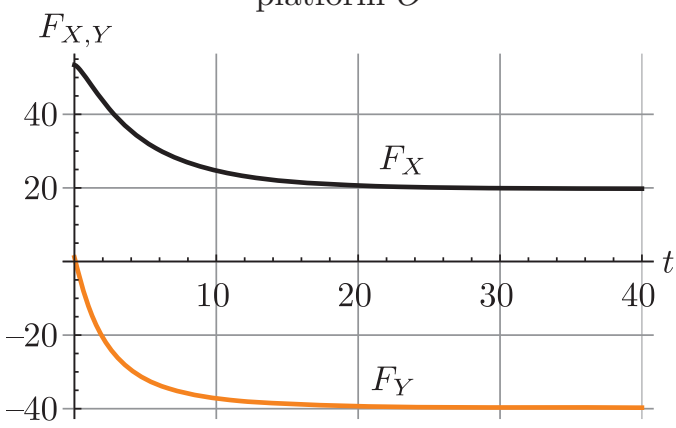

(c) Dependences $F_{X}(t)$ and $F_{Y}(t)$

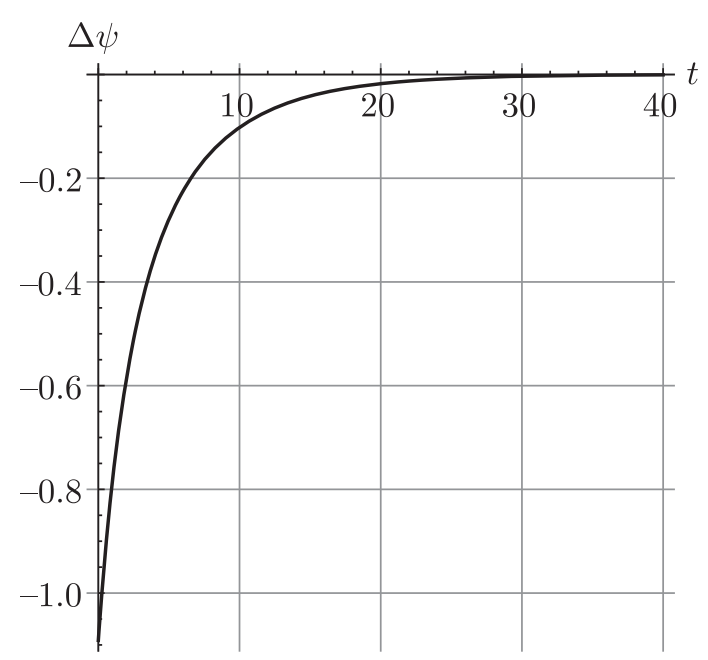

(b) Deviation $\Delta \psi$ from the steady rotation of the platform in the angle $\psi$

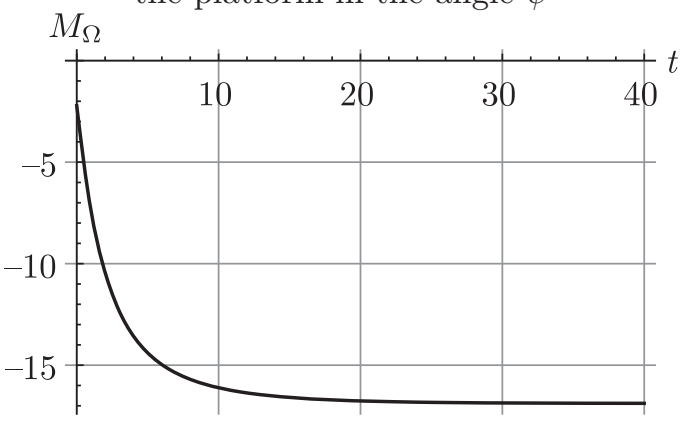

(d) Dependence $M_{\Omega}(t)$

Fig. 4. Implementation of the program motion of the point of the mobile platform $B$. The motion is consistent with additional constraints (stable steady rotation).

or, making use of the formula (4.5) for $\widetilde{V}_{B}$,

$$
\varepsilon \frac{d^{2} \widetilde{\psi}}{d \tau^{2}}+\{1+\varepsilon K \sin (\widetilde{\psi}+\beta)\} \frac{d \widetilde{\psi}}{d \tau}+s_{\omega}\left\{1-\frac{R_{B}}{\widetilde{R}_{B}} \cos (\widetilde{\psi}+\alpha)\right\}=0
$$

where $K=R_{B} \sqrt{\left(\varkappa_{11}-\varkappa_{21}\right)^{2}+\left(\varkappa_{12}-\varkappa_{22}\right)^{2}}$.

We rewrite the last equation in the form of the singularly perturbed system

$$
\left\{\begin{array}{l}
\frac{d \widetilde{\psi}}{d \tau}=\widetilde{\omega} \\
\varepsilon \frac{d \widetilde{\omega}}{d \tau}+\{1+\varepsilon K \sin (\widetilde{\psi}+\beta)\} \widetilde{\omega}+s_{\omega}\left\{1-\frac{R_{B}}{\widetilde{R}_{B}} \cos (\widetilde{\psi}+\alpha)\right\}=0 .
\end{array}\right.
$$


The degenerate system has the form

$$
\left\{\begin{array}{l}
\frac{d \widetilde{\psi}}{d \tau}=\widetilde{\omega} \\
\widetilde{\omega}+s_{\omega}\left\{1-\frac{R_{B}}{\widetilde{R}_{B}} \cos (\widetilde{\psi}+\alpha)\right\}=0 .
\end{array}\right.
$$

Let us find out if the transition to the degenerate problem is "correct". We consider the associated problem. We introduce the "fast" time $\tau_{\varepsilon}=\tau / \varepsilon$, and the system (4.10) takes the form

$$
\left\{\begin{array}{l}
\frac{d \widetilde{\psi}}{d \tau_{\varepsilon}}=\varepsilon \widetilde{\omega} \\
\frac{d \widetilde{\omega}}{d \tau_{\varepsilon}}+\{1+\varepsilon K \sin (\widetilde{\psi}+\beta)\} \widetilde{\omega}+s_{\omega}\left\{1-\frac{R_{B}}{\widetilde{R}_{B}} \cos (\widetilde{\psi}+\alpha)\right\}=0 .
\end{array}\right.
$$

At $\varepsilon=0$ we have $\widetilde{\psi}=$ const and

$$
\frac{d \widetilde{\omega}}{d \tau_{\varepsilon}}+\widetilde{\omega}+s_{\omega}\left\{1-\frac{R_{B}}{\widetilde{R}_{B}} \cos (\widetilde{\psi}+\alpha)\right\}=0 .
$$

Due to (4.13), the solution manifold for the degenerate system (4.11) is asymptotically stable in the "fast" time. By the Tikhonov theorem [24, 25], the "correctness" of using solutions of the degenerate system (4.11) for the approximate description of the behavior of the system (4.9) on the finite time interval is therefore proved.

We investigate the dynamics of the controlled motion of the system by analyzing the solution for the degenerate system (4.11). We rewrite it in the form

$$
\dot{\widetilde{\psi}}=\omega_{B}(\varrho \cos (\widetilde{\psi}+\alpha)-1)
$$

where $\varrho=R_{B} / \widetilde{R}_{B}$.

Figure 5 shows phase portraits of the angular motions satisfying Eq. (4.4), and the cumulative curve of the solutions of the degenerate system (4.11). For $R_{B}>\widetilde{R}_{B}$ there exist stable and unstable families of steady motions, which are represented in Fig. 5a as points of intersection of the cumulative curve of the degenerate problem with the axis $\Omega-\omega_{B}=0$. In the critical case, at $R_{B}=\widetilde{R}_{B}$, steady motions have a stable-unstable character. In Fig. 5b, contact points of the cumulative curve of the degenerate problem and of the straight line $\Omega-\omega_{B}=0$ correspond to such motions. For $R_{B}<\widetilde{R}_{B}$ there are no steady rotations and the platform executes rotational motion (Fig. 5c).

Let us consider the case of rotational motion in detail.

We rewrite Eq. (4.14) as

$$
\frac{d \widetilde{\psi}}{\varrho \cos (\widetilde{\psi}+\alpha)-1}=\omega_{B} d t
$$

For $R_{B}<\widetilde{R}_{B}(\varrho<1)$, the integration of the left-hand side of Eq. (4.15) yields [7, p. 162]

$$
\int \frac{d \widetilde{\psi}}{\varrho \cos (\widetilde{\psi}+\alpha)-1}=-\frac{2}{\sqrt{1-\varrho^{2}}} \operatorname{arctg} \frac{\sqrt{1-\varrho^{2}} \operatorname{tg} \frac{\widetilde{\psi}+\alpha}{2}}{1-\varrho},
$$




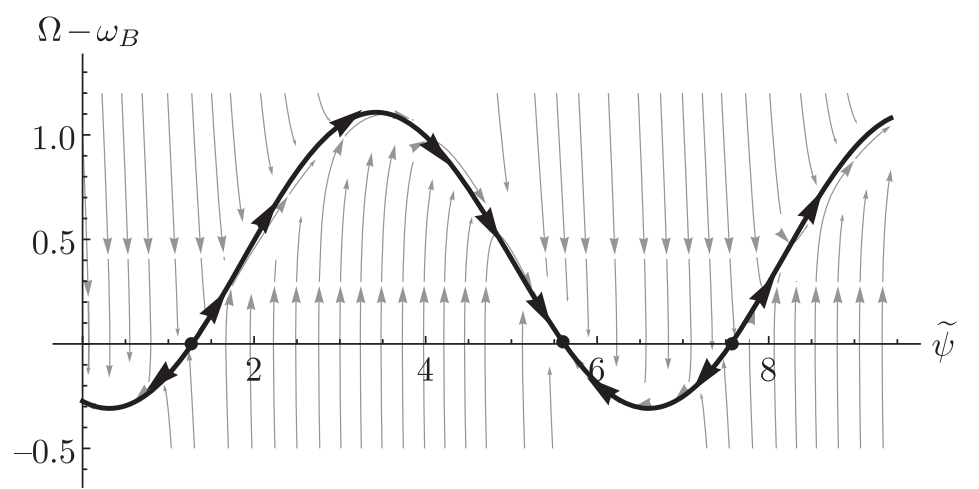

(a) $R_{B}>\widetilde{R}_{B}$

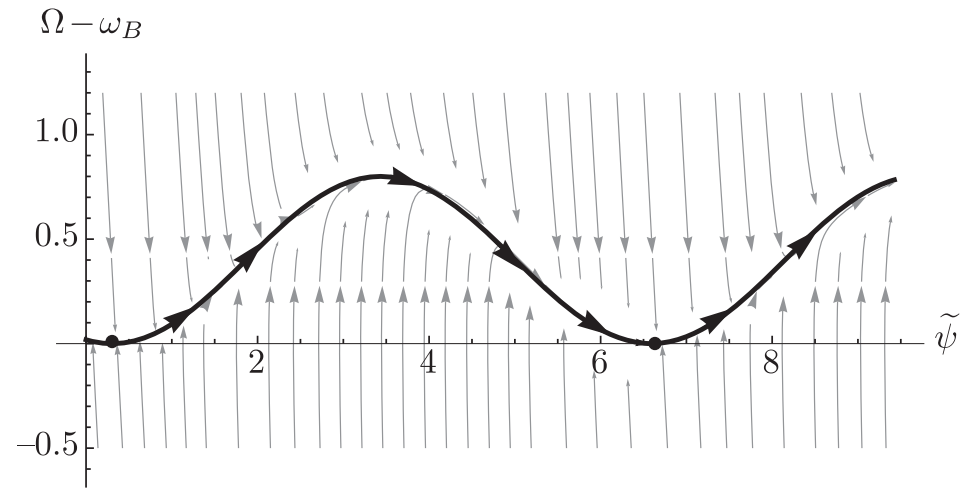

(b) $R_{B}=\widetilde{R}_{B}$

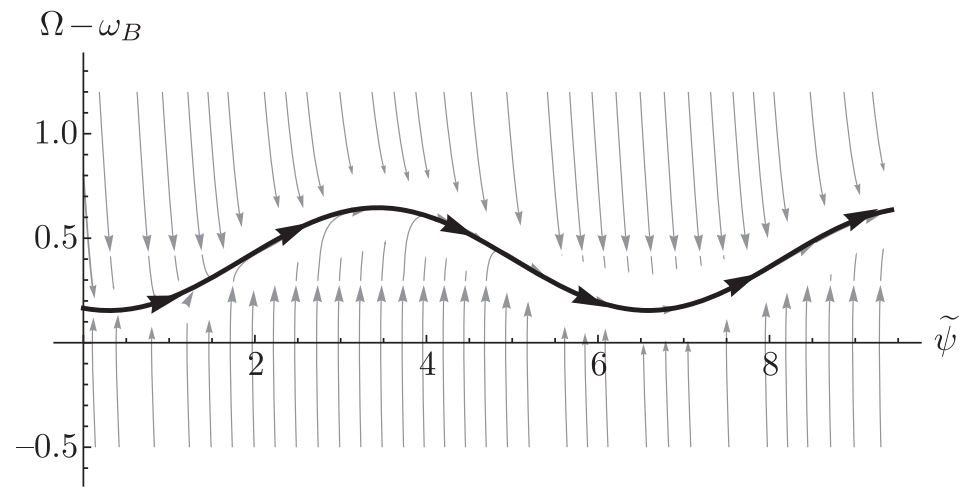

(c) $R_{B}<\widetilde{R}_{B}$

Fig. 5. Phase portraits of the angular motions of the platform during the uniform program motion of point $B$ in a circle for different values of its radius $R_{B}$.

from which, by passing to definite integrals, we obtain

$$
\begin{gathered}
\operatorname{tg} \frac{\widetilde{\psi}(t)+\alpha}{2}=-\sqrt{\frac{1-\varrho}{1+\varrho}} \operatorname{tg}\left(\frac{\sqrt{1-\varrho^{2}}}{2} \omega_{B} t-\frac{C_{1}}{2}\right), \\
C_{1}=2 \operatorname{arctg}\left(\sqrt{\frac{1+\varrho}{1-\varrho}} \operatorname{tg} \frac{\widetilde{\psi}(0)+\alpha}{2}\right) .
\end{gathered}
$$


Using the expressions of basic trigonometric functions in terms of the tangent of the halfangle, we find

$$
\cos (\widetilde{\psi}+\alpha)=\frac{\varrho+\cos \left(\sqrt{1-\varrho^{2}} \omega_{B} t-C_{1}\right)}{1+\varrho \cos \left(\sqrt{1-\varrho^{2}} \omega_{B} t-C_{1}\right)}
$$

and by virtue of (4.15) we obtain the time dependence of the deviation of the angular velocity of the platform

$$
\dot{\widetilde{\psi}}=\frac{\left(\varrho^{2}-1\right) \omega_{B}}{1+\varrho \cos \left(\sqrt{1-\varrho^{2}} \omega_{B} t-C_{1}\right)}
$$

in the asymptotic case.

The analysis of the last relation shows that, in the degenerate case, for $R_{B}<\widetilde{R}_{B}$, the angular velocity of the platform $\Omega=\dot{\tilde{\psi}}+\omega_{B}$ is a periodic function of time with a nonvanishing average, which tells us about the rotary character of the platform rotation. As the radius of the trajectory $R_{B}$ increases to the critical $\widetilde{R}_{B}, \varrho \rightarrow 1$ and the oscillation frequency of the angular velocity $\Omega$, which equals $\left|\omega_{B}\right| \sqrt{1-\varrho^{2}}$, tends to zero, and the amplitude increases to $\left|\omega_{B}\right|$. Here, at values of $\varrho$ close to unity, $\Omega$ is close to $\omega_{B}$ most part of the time, and the platform sharply makes a complete turn once per period. The above properties are illustrated in Fig. 6, which presents the results of numerical solution of the equation for the angular coordinate (4.3) obtained for $\omega_{B}=-0.4 \mathrm{~s}^{-1}, \varphi_{B}=90^{\circ}, b_{X}=0.4 \mathrm{~m}, b_{Y}=0.05 \mathrm{~m}\left(\widetilde{R}_{B}=0.65 \mathrm{~m}\right)$.

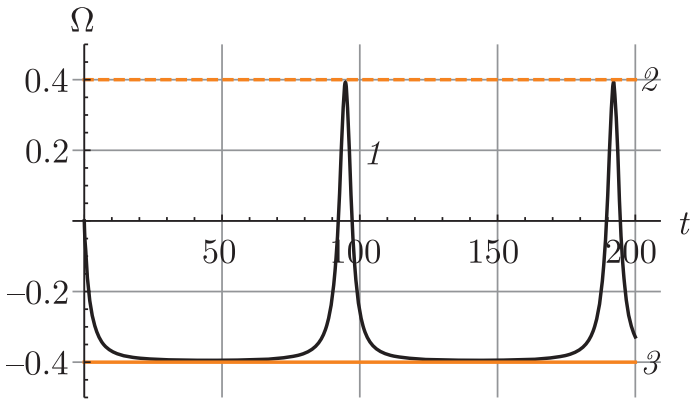

(a) $R_{B}=0.64 \mathrm{~m}$

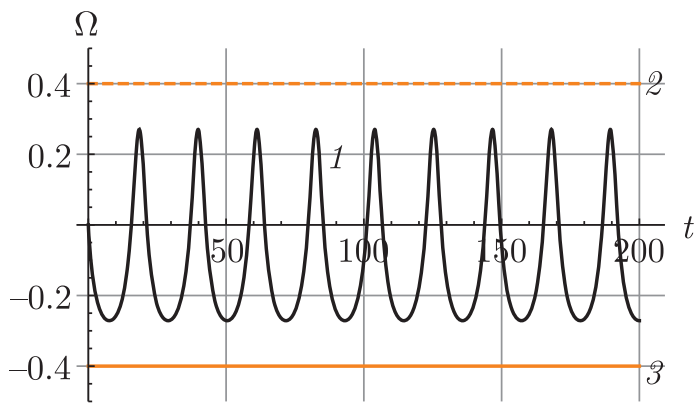

(c) $R_{B}=0.44 \mathrm{~m}$

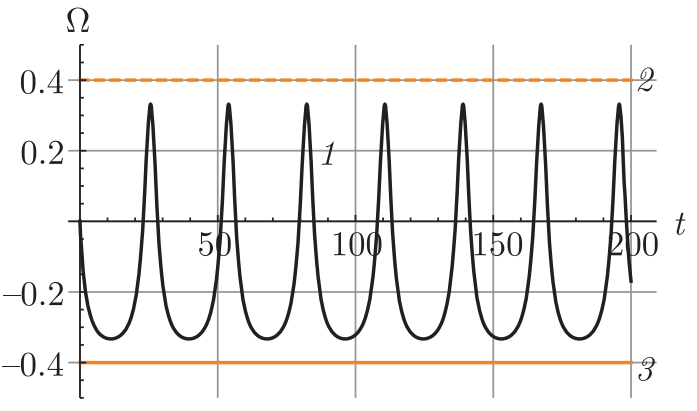

(b) $R_{B}=0.54 \mathrm{~m}$

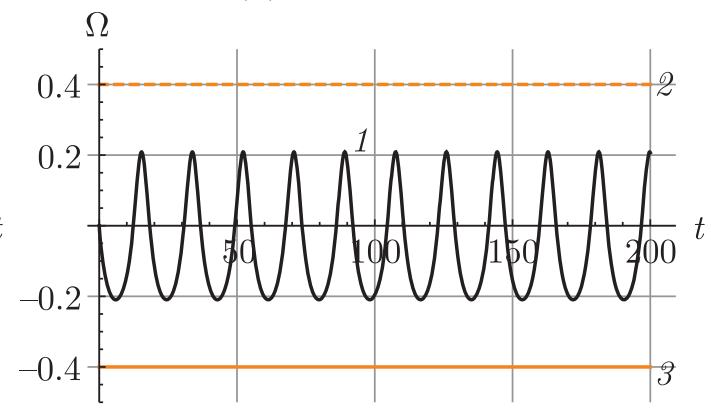

(d) $R_{B}=0.34 \mathrm{~m}$

$$
1-\Omega(t) ; 2-\omega_{B} ; 3-\left(-\omega_{B}\right)
$$

Fig. 6. The angular velocity of the platform $\Omega$ during the uniform program motion of point $B$ in a circle for different values of radius $R_{B}$. 
Figure 7 shows the trajectories of the points of the mobile platform plotted as a result of the numerical solution of Eq. (4.3). The resonance cases are considered: the values $R_{B}<\widetilde{R}_{B}$ are such that the variation frequencies of the angular velocity $\Omega$ and $\left|\omega_{B}\right|$ are related as natural numbers, that is,

$$
\sqrt{1-\varrho^{2}}=q, \quad q \in \mathbb{Q}_{+} .
$$

Here $R_{B}=\widetilde{R}_{B} \sqrt{1-q^{2}} ; \varphi_{B}=0$ with other parameter values being equal.

Let us consider a critical case. Since $[7$, p. 163$]$ for $R_{B}=\widetilde{R}_{B}(\varrho=1)$

$$
\int \frac{d \widetilde{\psi}}{\varrho \cos (\widetilde{\psi}+\alpha)-1}=\operatorname{ctg} \frac{\widetilde{\psi}+\alpha}{2},
$$

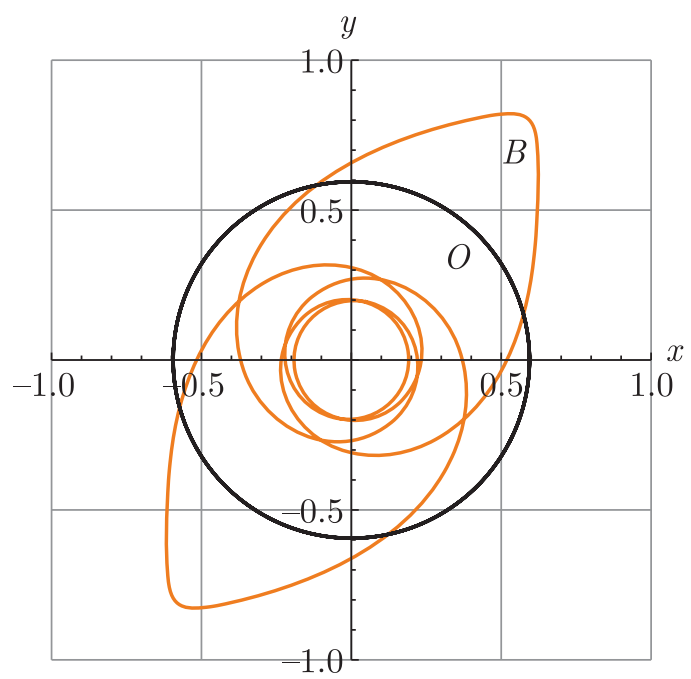

(a) $q=2 / 5, \quad R_{B}=0.5943 \mathrm{~m}$

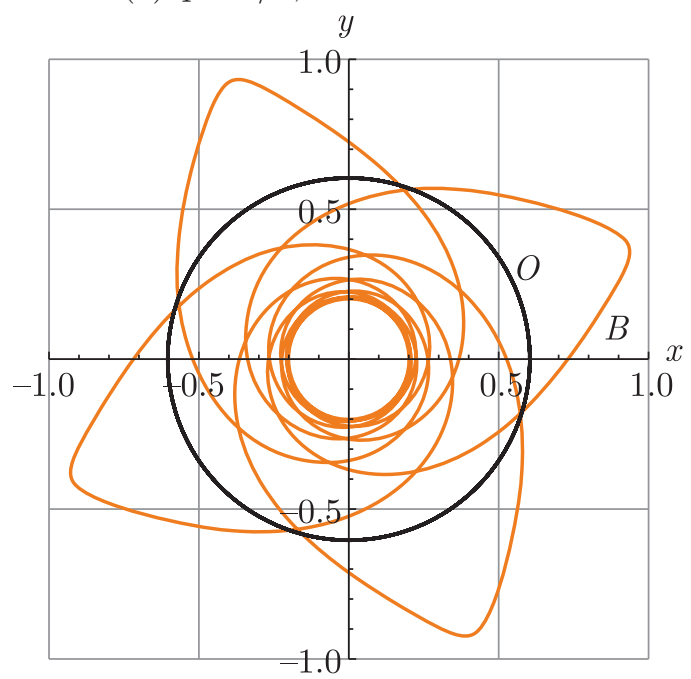

(c) $q=4 / 11, \quad R_{B}=0.6041 \mathrm{~m}$

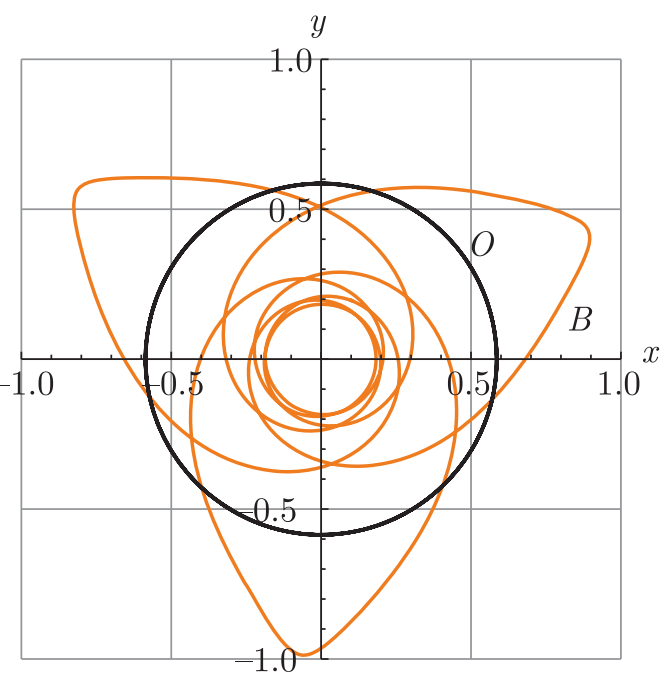

(b) $q=3 / 7, \quad R_{B}=0.5859 \mathrm{~m}$

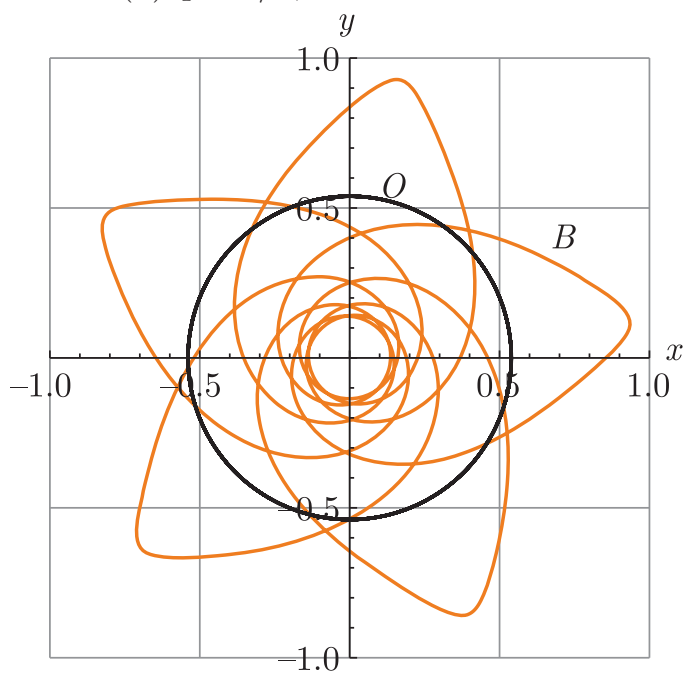

(d) $q=5 / 9, \quad R_{B}=0.5392 \mathrm{~m}$

Fig. 7. Trajectories of the center of the platform, $O$, during the uniform program motion of point $B$ in a circle for different values of radius $R_{B}$. 
integrating Eq. (4.15) yields

$$
\operatorname{ctg} \frac{\widetilde{\psi}(t)+\alpha}{2}=\omega_{B} t+\operatorname{ctg} \frac{\widetilde{\psi}(0)+\alpha}{2},
$$

whence

$$
\widetilde{\psi}(t)=2 \operatorname{arcctg}\left(\omega_{B} t+\operatorname{ctg} \frac{\widetilde{\psi}(0)+\alpha}{2}\right)-\alpha .
$$

In the case at hand, Eq. (4.15) has a family of singular solutions

$$
\widetilde{\psi}(t) \equiv \widetilde{\psi}_{s}[k]=-\alpha+2 \pi k, \quad k \in \mathbb{Z} .
$$

The analysis of the general solution obtained shows that, if $\omega_{B}<0$, then for the initial condition $\widetilde{\psi}(0)=\widetilde{\psi}_{s}[k]-0$ as $t \rightarrow \infty, \widetilde{\psi}(t) \rightarrow \widetilde{\psi}_{s}[k]$, and for $\widetilde{\psi}(0)=\widetilde{\psi}_{s}[k]+0$ as $t \rightarrow \infty$, $\widetilde{\psi}(t) \rightarrow \widetilde{\psi}_{s}[k+1]$. In the case $\omega_{B}>0$, for the initial condition $\widetilde{\psi}(0)=\widetilde{\psi}_{s}[k]-0$ as $t \rightarrow \infty$, $\widetilde{\psi}(t) \rightarrow \widetilde{\psi}_{s}[k-1]$, and for $\widetilde{\psi}(0)=\widetilde{\psi}_{s}[k]+0$ as $t \rightarrow \infty, \widetilde{\psi}(t) \rightarrow \widetilde{\psi}_{s}[k]$. The above properties of the solution of the degenerate problem allow us to conclude that the character of steady rotations is stable-unstable.

Let us illustrate the conclusions obtained with the results of modeling. Figure 8 shows the trajectories of the points of the platform for $\omega_{B}=-0.4 \mathrm{~s}^{-1}, \varphi_{B}=90^{\circ}, b_{X}=0.4 \mathrm{~m}$, $b_{Y}=0.05 \mathrm{~m}, R_{B}=\widetilde{R}_{B}=0.6485 \mathrm{~m}$ for two initial conditions of $\widetilde{\psi}(0)$, close to $\widetilde{\psi}_{s}=-0.10$. Here, when $\widetilde{\psi}(0)=0.0$, the platform makes an additional turn, and the trajectory of its center has a segment in the form of a lobe (curve 2).

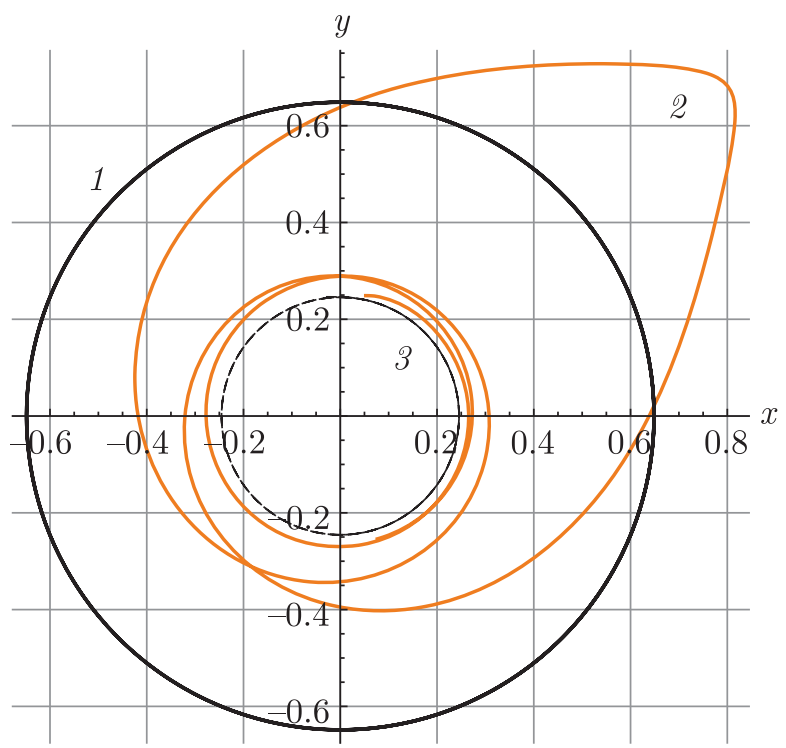

Trajectories: 1 - of point $B$

2 - of the center of the platform $O$ for $\widetilde{\psi}(0)=0.0$;

3 - of the center of the platform $O$ for $\widetilde{\psi}(0)=-0.2$.

Fig. 8. Trajectories of the center of the platform $O$ during the uniform program motion of point $B$ in a circle for $R_{B}=\widetilde{R}_{B}$. 
We proceed now to the case of "large" radii. For $R_{B}>\widetilde{R}_{B}(\varrho>1)$, the formula (4.18) takes the form

$$
\begin{aligned}
& \dot{\widetilde{\psi}}=\frac{\left(\varrho^{2}-1\right) \omega_{B}}{1 \pm \varrho \operatorname{ch}\left(\sqrt{\varrho^{2}-1} \omega_{B} t+\operatorname{Re} C_{2}\right)} \\
& C_{2}=2 \operatorname{arth}\left(\sqrt{\frac{\varrho+1}{\varrho-1}} \operatorname{tg} \frac{\widetilde{\psi}(0)+\alpha}{2}\right) .
\end{aligned}
$$

Here, the minus sign in the term of fraction is put if the argument of the inverse hyperbolic tangent exceeds unity in absolute value, and the constant $C_{2}$ has an imaginary part.

The analysis of the formula (4.19) shows that $\dot{\widetilde{\psi}}(t)$ decays as $\exp \left(-\sqrt{\varrho^{2}-1}\left|\omega_{B}\right| t\right)$, and the angular velocity $\Omega$ is set to the value $\omega_{B}$.

In the case considered, Eq. (4.15) has two families of singular solutions

$$
\begin{aligned}
& \widetilde{\psi}(t) \equiv \widetilde{\psi}_{s 1}[k]=\arccos \frac{1}{\varrho}-\alpha+2 \pi k, \\
& \widetilde{\psi}(t) \equiv \widetilde{\psi}_{s 2}[k]=-\arccos \frac{1}{\varrho}-\alpha+2 \pi k,
\end{aligned} \quad k \in \mathbb{Z},
$$

corresponding to the steady rotations of the platform with $\Omega=\omega_{B}$.

Under the assumptions made concerning the parameter values, one of these families is stable, and the other is unstable (depending on the sign of $\omega_{B}$ ). In the case where $\omega_{B}$ is large and the parameter $\varepsilon=\left|\omega_{B}\right| / \xi_{3}$ cannot be considered small, both families of steady rotations may be unstable (see Fig. 3).

\subsection{The case of singular position of the point moving in a circle on the platform}

Previously, in the analysis of the angular motion of the platform (4.3) it was assumed that the denominators of the expressions (4.5) defining $\widetilde{R}_{B}$ and $\widetilde{V}_{B}$ do not equal zero. Let us consider the case where

$$
\xi_{1}+\varkappa_{22} \omega_{B}=0, \quad \xi_{2}-\varkappa_{21} \omega_{B}=0
$$

and the denominator in the formula for $\widetilde{R}_{B}$ vanishes.

In accordance with the expressions (3.10), the last conditions are equivalent to the following system of equations:

$$
\zeta_{Y} b_{X}+\omega_{B}\left(m_{s} b_{Y}-m a_{Y}\right)=0, \quad \zeta_{X} b_{Y}-\omega_{B}\left(m_{s} b_{X}-m a_{X}\right)=0,
$$

the solution of which has the form

$$
b_{X}=\frac{m \omega_{B}\left(m_{s} \omega_{B} a_{X}+\zeta_{X} a_{Y}\right)}{\zeta_{X} \zeta_{Y}+m_{s}^{2} \omega_{B}^{2}}, \quad b_{Y}=\frac{m \omega_{B}\left(m_{s} \omega_{B} a_{Y}-\zeta_{Y} a_{X}\right)}{\zeta_{X} \zeta_{Y}+m_{s}^{2} \omega_{B}^{2}} .
$$

Substituting numerical values of the parameters (2.17) into the formula (4.20) yields

$$
b_{X}=0.09 \mathrm{~cm}, \quad b_{Y}=0.1 \mathrm{~cm} .
$$

RUSSIAN JOURNAL OF NONLINEAR DYNAMICS, 2018, 14(2), 265-290 
For the above values of the parameters $b_{X}$ and $b_{Y}$ the constant $\widetilde{V}_{B}=2.9 \mathrm{~km} / \mathrm{s}$. Such a large value of $\widetilde{V}_{B}$ is explained by the fact that the denominator in the expression (4.5) for $\widetilde{V}_{B}$ vanishes at $b_{X}=b_{Y}=0$.

Therefore, if the formulae (4.1) define the program motion of point $B$ with the coordinates (4.20), then some nonlinear terms in Eq. (4.3) vanish, and other terms become negligible due to the large value of $\widetilde{V}_{B}$. Taking into account the above properties, the equation of angular motions (4.3) takes the form

$$
\ddot{\widetilde{\psi}}+\xi_{3}\left(\dot{\widetilde{\psi}}+\omega_{B}\right)=0
$$

or, taking into account that $\dot{\widetilde{\psi}}=\Omega-\omega_{B}$,

$$
\dot{\Omega}+\xi_{3} \Omega=0 .
$$

The analysis of the last relation allows us to draw a conclusion about the decay of the angular velocity $\Omega$ and the onset of translational motion of the platform when implementing the program motion (4.1) for the point with the coordinates (4.20). The asymptotic solution $\Omega(t) \equiv 0$ can also be obtained by solving the degenerate problem (4.15) with $\varrho=0$.

Let us illustrate this conclusion by mathematical modeling of the motion of the mobile youBot robot. The program motion (4.1) is characterized by the parameters $\omega_{B}=-0.4 \mathrm{~s}^{-1}$, $R_{B}=0.7 \mathrm{~m}, \varphi_{B}=90^{\circ}$. The abscissa and the ordinate of point $B$ in the plane $O X Y$ are given by the formulae (4.20). At the initial instant $\psi(0)=0, \Omega(0)=0$.

The angular velocity of the platform obeys Eq. (4.4), and the generalized control forces $F_{X}$, $F_{Y}$ and $M_{\Omega}$ are calculated by the formulae (3.11)-(3.13).

The results of the numerical modeling of the motion considered are presented in Fig. 9. The conclusions about the translational character of motion accurately describe the modeling results, namely, that the values of the angular velocity $\Omega$ are negligible (see Fig. 9b).

\section{Analysis of power inputs needed to control the platform}

The results of numerical investigation of the control algorithm (3.9), (3.11)-(3.13) show its optimality and suboptimality from the viewpoint of the work quantity of generalized control forces

$$
A_{\mathfrak{C}}(t)=\int_{0}^{t} \dot{\pi}^{\mathrm{T}}(\tau) \boldsymbol{\Pi}_{\mathfrak{C}}(\tau) d \tau=\int_{0}^{t}\left[V_{X}(\tau) F_{X}(\tau)+V_{Y}(\tau) F_{Y}(\tau)+\Omega(\tau) M_{\Omega}(\tau)\right] d \tau .
$$

On the one hand, the above property is due to the fact that by the law (3.9), (3.11)-(3.13) only two degrees of freedom (which are translational) are regulated, and the platform's rotation is inertial motion. On the other hand, in this case the structure of the control actions repeats the structure of the reactions of the ideal constraints the equations of which are identical with the equations of program motion (3.2). The extreme properties of the constraint reactions and their work in deviations from the released motion, resulting from the laws of mechanics, make the above method optimal in a sense [15].

We present the results of calculation of the work of the control forces $A_{\mathfrak{C}}$ according to the results of mathematical modeling for the motion of the mobile youBot robot. The program motion of point $B(4.1)$ in a circle is characterized by the parameters $\omega_{B}=-0.4 \mathrm{~s}^{-1}, R_{B}=0.7 \mathrm{~m}$, $\varphi_{B}=90^{\circ}$. The abscissa and the ordinate of $B$ in the plane $O X Y$ are equal to $b_{X}=0.4 \mathrm{~m}$, $b_{Y}=0.05 \mathrm{~m}$. 


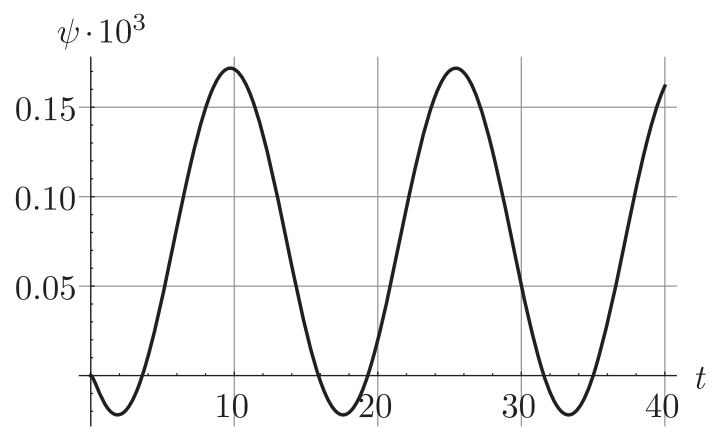

(a) Rotation angle of the platform $\psi(t)$

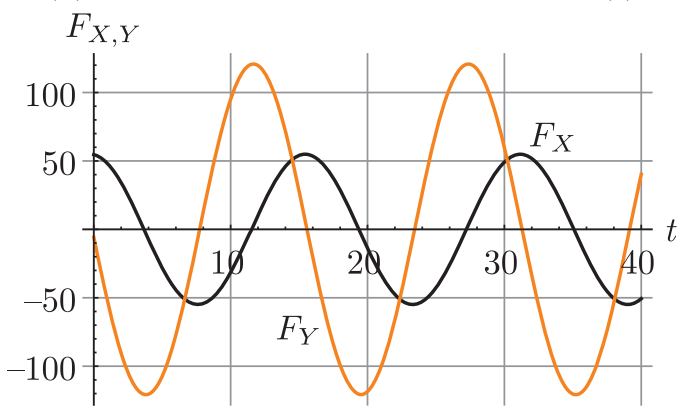

(c) Dependences $F_{X}(t)$ and $F_{Y}(t)$

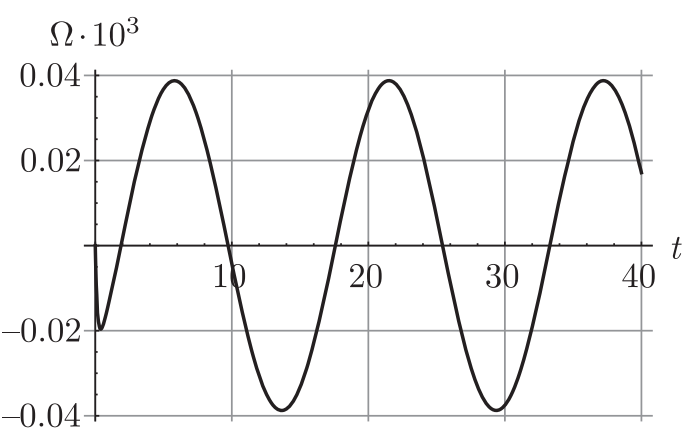

(b) Angular velocity of the platform $\Omega(t)$

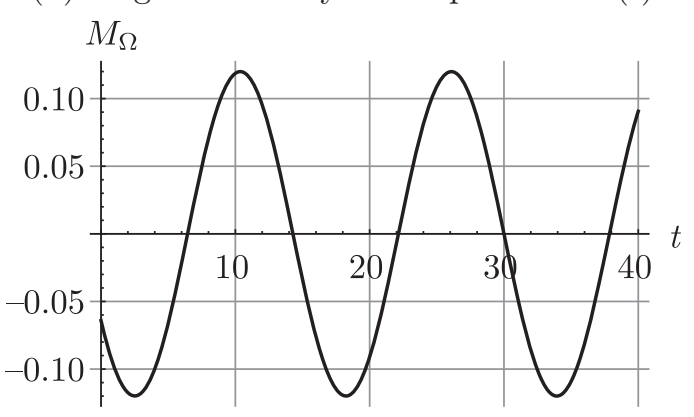

(d) Dependence $M_{\Omega}(t)$

Fig. 9. Implementation of the program motion of the point of the mobile platform $B$. The motion is consistent with the additional constraints (the motion which is close to translational motion).

Let us consider several methods for controlling the platform:

- The angular velocity of the platform obeys Eq. (4.4), and the generalized control forces are calculated by the formulae (3.11)-(3.13). At the initial instant $\psi(0)=0, \psi^{\prime}(0)=0$. The plots for the generalized control forces are shown in Fig. 4.

- The angular motion of the platform is redefined by the relation $\psi^{\prime}(t)=$ const (the initial angle $\psi(0)$ is arbitrary). The computation of the control forces is carried out by substituting the known laws of variations of coordinates, velocities and accelerations into the equations of motion (2.13)-(2.15). For the particular case of translational motion $\left(\psi^{\prime}(t)=0\right.$, $\psi(0)=0)$ the plots of the generalized control forces accurately repeat the ones shown in Fig. 9.

The relevant plots of variation in the work of the control forces $A_{\mathfrak{C}}$ are shown in Fig. 10. From the viewpoint of the quantity of the power inputs required for the control of the platform, the control law (4.4), (3.11)-(3.13) is the most preferable of all the laws considered.

Note that the results of modeling (Fig. 10) show that the motion of point $B$ of the platform in a circle at $\psi=\omega_{B} t$ requires less power than the "backward" motion where $\psi=\omega_{B} t+180^{\circ}$.

Indeed, if $\psi(t)=\omega_{B} t+\psi(0)$ and $\varphi_{B}=90^{\circ}$, then by the formulae (3.6) and (4.1) the velocity components of the center of the platform are

$$
V_{X}=\omega_{B}\left(b_{Y}-R_{B} \cos \psi(0)\right), \quad V_{Y}=\omega_{B}\left(R_{B} \sin \psi(0)-b_{X}\right) .
$$

When $\psi(0)=0$, the longitudinal velocity is $\left|V_{X}\right|=\left|\omega_{B}\left(R_{B}-b_{Y}\right)\right|$, and this is less than its value $\left|V_{X}\right|=\left|\omega_{B}\left(b_{Y}+R_{B}\right)\right|$ when $\psi(0)=180^{\circ}$ (the values of $V_{Y}$ are in both cases the same, 


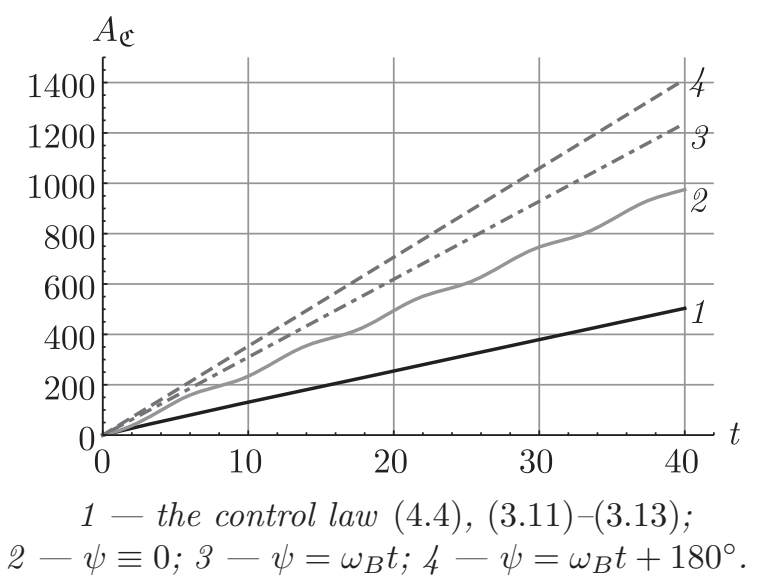

Fig. 10. Work of the control torques during the motion of the platform in a circle.

$\left.b_{Y}>0\right)$. Since overcoming the linear viscous friction makes the main contribution to the work of the control forces, in the former case the power inputs into the motion will be lower than in the latter case (see Fig. 10, curves 3 and 4, respectively).

\section{Conclusions}

This paper solves the problem of implementing the program motion of an arbitrary point on the mobile omnidirectional platform youBot. The structure of the control forces repeats the structure of the reactions of ideal constraints with which the program motion is consistent.

The uniform motion of a point of the platform in a circle is presented to show that, depending on the parameters of the program motion, the rotation of the platform can be different. Steady motions occur if the radius of the circle exceeds some critical value. Under certain conditions all steady regimes are unstable. For the radii of the circle which are smaller than the critical one, the angular motion of the platform is rotational.

Conditions are found under which the platform's behavior is intuitively predictable: it executes translational motion or stable steady rotations with angular velocity equal to the cyclic frequency of motion of the point of the platform in a circle.

"Large" values of the reduced coefficients of viscous friction in the joints of the bodies have allowed us to single out a small parameter at a higher derivative. The correctness of passing to a degenerate problem is shown in the analysis of the platform's rotation. An analytical solution of the degenerate problem is constructed for the equation of angular motions at different values of the radius of the program trajectory.

The results obtained allow planning of the motion of a mobile robot, namely, specifying the position of the point moving in a circle on the platform, and selecting the radius of the trajectory and the speed of going round. The asymptotic solution to the equation of controlled dynamics can be used for an analytic study of the power inputs required for the implementation of the program motion, and for their optimization.

\section{Acknowledgments}

The author extends his gratitude to Professor A. I. Kobrin for fruitful discussions and useful comments on this paper. 


\section{References}

[1] Adamov, B. I. and Kobrin, A. I., Parametric Identification of the Mathematical Model of the Omnidirectional Mobile Robot KUKA youBot, Mekhatronika, Avtomatizatsiya, Upravlenie, 2018, vol. 19, no. 4, pp. 251-258 (Russian).

[2] Adamov, B. I. and Orlov, I. V., Control of a Mobile Manipulator in Cylindrical Coordinate System, Vestn. Mosk. Energ. Inst., 2012, no. 1, pp. 28-35 (Russian).

[3] Beghin H. Étude théorique des compas gyrostatiques Anschütz et Sperri: Thése, Paris: Faculté des sciences de Paris, 1922.

[4] Borisov, A. V., Kilin, A. A., and Mamaev, I.S., An Omni-Wheel Vehicle on a Plane and a Sphere, Nelin. Dinam., 2011, vol. 7, no. 4, pp. 785-801 (Russian).

[5] Gantmacher, F. R., The Theory of Matrices: Vols. 1, 2, New York: Chelsea, 1959.

[6] Golubev, Yu. F., Appel's Function in the Dynamics of Rigid Body Systems, Preprint No. 49, Moscow: KIAM, 2014 (Russian).

[7] Gradshtein, I. S. and Ryzhik, I. M., Table of Integrals, Series, and Products, 7th ed., Amsterdam: Acad. Press, 2007.

[8] Zobova, A. A., Application of Laconic Forms of the Equations of Motion in the Dynamics of Nonholonomic Mobile Robots, Nelin. Dinam., 2011, vol. 7, no. 4, pp.771-783 (Russian).

[9] Kilin, A. A. and Bobykin, A. D., Control of a Vehicle with Omniwheels on a Plane, Nelin. Dinam., 2014, vol. 10, no. 4, pp. 473-481 (Russian).

[10] Kilin A. A., Karavaev Yu. L., Klekovkin A. V. Kinematic Control of a High Manoeuvrable Mobile Spherical Robot with Internal Omni-Wheeled Platform, Nelin. Dinam., 2014, vol.10, no. 1, pp. 113-126 (Russian).

[11] Kozlov, V.V., Principles of Dynamics and Servo-Constraints, Nelin. Dinam., 2015, vol.11, no. 1, pp. 169-178 (Russian).

[12] Kozlov, V.V., The Dynamics of Systems with Servoconstraints: 1, Regul. Chaotic Dyn., 2015, vol. 20, no. 3, pp. 205-224.

[13] Kozlov, V. V., The Dynamics of Systems with Servoconstraints: 2, Regul. Chaotic Dyn., 2015, vol. 20, no. 4, pp. 401-427.

[14] Markeev, A.P., Theoretical Mechanics, Izhevsk: R\&C Dynamics, Institute of Computer Science, 2007 (Russian).

[15] Markeev, A. P., On the Gauss Principle, in Collection of Articles on Theoretical Mechanics: Vol. 23, A. N. Kolesnikov (Ed.), Moscow: Mosk. Gos. Univ., 2000, pp. 29-44 (Russian).

[16] Martynenko, Yu. G., Motion Control of Mobile Wheeled Robots, J. Math. Sci. (N. Y.), 2007, vol. 147, no. 2, pp.6569-6606; see also: Fundam. Prikl. Mat., 2005, vol.11, no.8, pp. 29-80.

[17] Martynenko, Yu. G., Orlov, I. V., Motion Control Software for Telescopic Manipulator on the Mobile Platform, Vestn. Mosk. Energ. Inst., 2003, no. 5, pp. 60-70 (Russian).

[18] Martynenko, Yu. G. and Formal'skii, A. M., On the Motion of a Mobile Robot with Roller-Carrying Wheels, J. Comput. Syst. Sci. Int., 2007, vol.46, no.6, pp.976-983; see also: Izv. Akad. Nauk. Teoriya i Sistemy Upravleniya, 2007, no. 6, pp. 142-149.

[19] Martynenko, Yu. G., Stability of Steady Motions of a Mobile Robot with Roller-Carrying Wheels and a Displaced Centre of Mass, J. Appl. Math. Mech., 2010, vol.74, no.4, pp.436-442; see also: Prikl. Mat. Mekh., 2010, vol. 74, no. 4, pp. 610-619.

[20] Mukhametzyanov, I. A., The Principle of Feedback on the Quasi-Accelerations for Unstressed Stabilization in Finite Time of Given Manifolds of Mechanical and Generalized Systems, Vestn. RUDN. Mat. Inform. Fiz., 2014, no. 3, pp. 107-114 (Russian).

[21] Mukharlyamov, R. G., Simulation of Control Processes, Stability and Stabilization of Systems with Program Constraints, J. Comput. Syst. Sci. Int., 2015, vol.54, no. 1, pp. 13-26; see also: Izv. Akad. Nauk. Teoriya i Sistemy Upravleniya, 2015, no. 1, pp. 15-28. 
[22] Mukharlyamov, R. G. and Abramov, N. V., Dynamic Control of Manipulator with Program Constraints, in Problems of Mechanics and Control: Nonlinear Dynamical Systems, No. 43, Perm: Perm State Univ., 2011, pp. 90-102 (Russian).

[23] Neimark, Ju. I. and Fufaev, N.A., Dynamics of Nonholonomic Systems, Trans. Math. Monogr., vol. 33, Providence, R.I.: AMS, 1972.

[24] Novozhilov, I. V., Fractional Analysis. Methods of Motion Decomposition, Basel: Birkhäuser, 1997.

[25] Tikhonov, A. N., Systems of Differential Equations Containing Small Parameters in the Derivatives, Mat. Sb. (N. S.), 1952, vol.31(73), no. 3, pp. 575-586 (Russian).

[26] Adascalitei, F. and Doroftei, I., Practical Applications for Mobile Robots Based on Mecanum Wheels: A Systematic Survey, The Romanian Review Precision Mechanics, Optics \& Mechatronics, 2011, no. 40, pp. 21-29.

[27] Gfrerrer, A., Geometry and Kinematics of the Mecanum Wheel, Comput. Aided Geom. Des., 2008, vol. 25, no. 9, pp. 784-791.

[28] Ilon, B.E., Wheels for a Course Stable Selfpropelling Vehicle Movable in Any Desired Direction on the Ground or Some Other Base: Patent Sweden B60B 19/12 (20060101); B60b 019/00, $\mathrm{REF} / 3876255$ (November 13, 1972).

[29] Indivery, G., Paulus, J., and Ploge, P.-G., Motion Control of Swedish Wheeled Mobile Robot in the Presence of Actuator Saturation, in RoboCup 2006: Robot Soccer World Cup X, G. Lakemeyer, E.Sklar, D. G. Sorrenti, T. Takahashi (Eds.), Lecture Notes in Comput. Sci., vol.4434, New York: Springer, 2007, pp. 29-44.

[30] Ivanov, A.P., On the Control of a Robot Ball Using Two Omniwheels, Regul. Chaotic Dyn., 2015, vol. 20, no. 4, pp. 441-448.

[31] Li, X. and Zell, A., Motion Control of an Omnidirectional Mobile Robot, in Informatics in Control, Automation and Robotics: Selected Papers from the International Conference on Informatics in Control, Automation and Robotics (2007): Part 2, J. Filipe, J. A. Cetto, J. L. Ferrier (Eds.), Lecture Notes in Electrical Engineering, vol. 24, Berlin: Springer, 2009, pp. 181-193.

[32] Lin, L.-C. and Shih, H.-Y., Modeling and Adaptive Control of an Omni-Mecanum-Wheeled Robot, Intelligent Control and Automation, 2013, vol. 4, no. 2, pp. 166-179.

[33] Planitz, M., Inconsistent Systems of Linear Equations, Math. Gazette, 1979, vol. 63, no. 425, pp. 181185.

[34] Plumpton, J. J., Hayes, M.J.D., Langlois, R. G., and Burlton, B. V., Atlas Motion Platform Mecanum Wheel Jacobian in the Velocity and Static Force Domains, in CCToMM Mechanisms, Machines, and Mechatronics Symposium, 2013, 10 p.

[35] Purwin, O. and D'Andrea, R., Trajectory Generation and Control for Four Wheeled Omnidirectional Vehicles, Robot. Auton. Syst., 2006, no. 54, no. 1, pp. 13-22.

[36] Zimmermann, K., Zeidis, I., and Behn, C., Mechanics of Terrestrial Locomotion: With a Focus on Non-pedal Motion Systems, London: Springer, 2010.

[37] http://www.youbot-store.com 